hep-th/0312314

MIT-CTP-3459

ITP-UH-36/03

\title{
String Field Theory Projectors for Fermions of Integral Weight
}

\author{
Matthias $\operatorname{Ihl}^{a}$, Alexander Kling ${ }^{a}$ and Sebastian Uhlmann ${ }^{b}$ \\ ${ }^{a}$ Institut für Theoretische Physik \\ Universität Hannover \\ 30167 Hannover, Germany \\ E-mail: msihl, kling@itp.uni-hannover.de \\ ${ }^{b}$ Center for Theoretical Physics \\ Massachusetts Institute of Technology \\ Cambridge, MA 02139, USA \\ E-mail: uhlmann@lns.mit.edu
}

Abstract:

The interaction vertex for a fermionic first order system of weights $(1,0)$ such as the twisted $b c$ system, the fermionic part of $\mathrm{N}=2$ string field theory and the auxiliary $\eta \xi$ system of $\mathrm{N}=1$ strings is formulated in the Moyal basis. In this basis, the Neumann matrices are diagonal; as usual, the eigenvectors are labeled by $\kappa \in \mathbb{R}$. Oscillators constructed from these eigenvectors make up two Clifford algebras for each nonzero value of $\kappa$. Using a generalization of the Moyal-Weyl map to the fermionic case, we classify all projectors of the star-algebra which factorize into projectors for each $\kappa$-subspace. At least for the case of squeezed states we recover the full set of bosonic projectors with this property. Among the subclass of ghost number-homogeneous squeezed state projectors, we find a single class of BPZ-real states parametrized by one (nearly) arbitrary function of $\kappa$. This class is shown to contain the generalized butterfly states. Furthermore, we elaborate on sufficient and necessary conditions which have to be fulfilled by our projectors in order to constitute surface states. As a byproduct we find that the full star product of $\mathrm{N}=2$ string field theory translates into a canonically normalized continuous tensor product of Moyal-Weyl products up to an overall normalization. The divergent factors arising from the translation to the continuous basis cancel between bosons and fermions in any even dimension. 


\section{Introduction}

Over the last years, open string field theory has provided evidence that it captures essential offshell string physics, namely the process of tachyon condensation on unstable D-brane systems (for reviews see $[1-5])$. This applies to cubic bosonic open string field theory [6] as well as to its supersymmetric generalization [7] and Berkovits' superstring field theory [8]. Most of this evidence relies heavily on numerical work. A fully satisfactory analytic solution to the equations of motion of open string field theory in any of its guises is currently missing.

In order to overcome this rather unsatisfactory situation, a version of open string field theory around the open bosonic string tachyon vacuum has been advocated in [9]. The key assumption in this proposal is the pure ghost nature of the kinetic operator reflecting the absence of physical open string excitations. In turn, this assumption leads to a factorization of the equations of motion into matter and ghost parts.

The matter part of these equations is a projector condition. An enormous amount of work has been done in the meantime to identify projectors of the star algebra in the matter sector [1020] and to describe their properties. Apart from the identity string field, the most prominent representatives are the sliver and the so-called butterfly. The latter ones belong to a one-parameter family of surface states dubbed generalized butterfly states.

The ghost part of the equations of motion remain complicated to solve (see, e.g., [21, 22]). However, projectors also appear in this context. In [23] an auxiliary boundary conformal field theory was introduced in order to construct solutions to the ghost equations of motion in terms of surface state projectors of the so-called twisted $b c$ system. This BCFT is obtained by twisting the energy momentum tensor with the derivative of the ghost number current. ${ }^{1}$

Eventually, we point out that projectors appear naturally in certain solution generating techniques proposed to solve the equations of motion of string field theory [27-31].

The subject of most of the references mentioned above are surface state projectors $[32,33]$. These are projectors with field configurations arising from path integrations over fixed Riemann surfaces whose boundary consists of a parametrized open string and a piece with open string boundary conditions. A very fundamental result in this realm [17] was that all such surfaces with the property that their boundaries touch the midpoint of the open string lead to projector functionals in the star algebra. Although this class of projectors is quite large, it is advantageous to look for projectors without these singular property which could eventually lead to D-brane solutions with finite energy densities.

This is the motivation for the present paper. We study projectors of a fermionic first order system of weights $(1,0)$. It is shown that projectors of this system give rise to bosonic projectors. Such a first order system appears in bosonic string field theory as the twisted $b c$ system, in the fermionic sector of $\mathrm{N}=2$ string field theory [34] and as the auxiliary $\eta \xi$ ghost system of $\mathrm{N}=1$ strings which is introduced in the process of fermionization of the superconformal ghosts [35]. We use a reformulation of the interaction vertex for such a system, which has been constructed explicitly in [36] (see also [37]), in terms of a continuous Moyal basis [38-44]. ${ }^{2}$ The diagonalization of the vertex is greatly simplified due to an intimate relation of the bosonic matter Neumann matrices and

\footnotetext{
${ }^{1}$ For a recent discussion based on a regulated version of the twisted butterfly state see [24] and in terms of a deformed sliver state see [25]. For an attempt to solve Witten's cubic open string field theory using the butterfly state see [26].

${ }^{2}$ The Witten vertex was first reformulated in terms of Moyal products in $[45,46]$ using a discrete basis. In this paper, we find it useful to work in the continuous Moyal basis which is equivalent to the discrete basis.
} 
those for the fermionic $(1,0)$-system $[36,37]$. In the diagonal basis, the star algebra decomposes into a product of infinitely many pairs of Clifford algebras labeled by a parameter $\kappa \in \mathbb{R}_{+}$. We employ a fermionic version of the Moyal-Weyl map known from noncommutative field theories to map the generators of these Clifford algebras to operators on some auxiliary Fock space; this enables us to determine all projectors in the star algebra which are already projectors for each value of $\kappa$. Among the general classes of projectors obtained by this procedure we find a single class of BPZ-real squeezed state projectors which are neutral w.r.t. the $U(1)$ current of the first order system. This class is naturally parametrized by a single odd and integrable function of the parameter $\kappa$ and contains the generalized butterfly states as a subclass. It is demonstrated how to determine surface states in this class. ${ }^{3}$ A condition on the maps (conventionally denoted by $f$ ) defining the shape of the surface is given.

As a byproduct we find that the full star product of $\mathrm{N}=2$ string field theory translates into a canonically normalized continuous tensor product of Moyal-Weyl products up to an overall normalization. The infinite factors arising from the translation to the continuous Moyal basis cancel between bosons and fermions in any even dimension. This is another direct consequence of the intimate relation between the bosonic and fermionic Neumann coefficients mentioned above and is similar to an analogous cancellation of anomalies observed in [36].

The paper is organized as follows: We start with a brief introduction to the fermionic first order system and its connection to the bosonic system in section 2. This relation can be used to immediately diagonalize the interaction vertex. In the diagonal basis, a comparison of the integral kernel of the star product with that of a canonical Clifford algebra product determines the (fermionic) Moyal-Weyl pairs. It is shown that the infinite factors from the translation to this basis cancel between bosons and fermions in $\mathrm{N}=2$ string field theory in any even dimension, in accordance with the central charge $c=-2$ of the first order system. In section 3, we apply the Moyal-Weyl map in the way described above and classify all projectors which are already projectors for each value of $\kappa$. The reality condition excludes some of these classes; again only a subset consists of squeezed state projectors. In section 4, we derive a condition on our coefficient functions such that the corresponding states are squeezed states. It turns out that there is only one class of real squeezed state projectors which are also neutral w.r.t. the $U(1)$ "ghost number" current of the first order system. It is demonstrated that the generalized butterfly states are contained in this class. An inverse transformation from string fields to operators in the auxiliary Fock space is given; it facilitates the computation of star products considerably. Moreover, from squeezed state projectors of this type one can recover the full set of bosonic projectors satisfying the same factorization properties. In section 5 , we outline the general method to determine whether a given squeezed state is a surface state. We find a condition on the map $f$ defining the surface state which is necessary in order for it to define a surface state projector with the above-mentioned factorization property. We then demonstrate the procedure in the case of the generalized butterfly states. Finally, we offer some concluding remarks in section 6 .

\section{The interaction vertex in Moyal form}

In this section we reformulate the interaction vertex of the fermionic first order system [36] in the continuous Moyal basis. General methods for the diagonalization of the fermionic vertex were presented in $[47,43,37]$. However, it seems advantageous to exploit the relation between the Neumann coefficients for the bosons and those for the fermionic system with weights $(1,0)$. To begin with we review the results for the bosonic Neumann coefficients.

\footnotetext{
${ }^{3}$ Under the proviso that the surface state projector factorizes into projectors for each value of $\kappa$, they all have to reside in this class.
} 
Diagonalization of the bosonic coefficients. The bosonic interaction vertex in momentum basis is given by $[48,49]$

$$
\left\langle V_{3}\right|=\int d^{D} p^{(1)} d^{D} p^{(2)} d^{D} p^{(3)} \delta^{D}\left(p^{(1)}+p^{(2)}+p^{(3)}\right)^{(123)}\langle p, 0| \exp [-V],
$$

where

$$
V=\frac{1}{2} \sum_{r, s} \sum_{m, n \geq 1} \eta_{\mu \nu} a_{m}^{(r) \mu} V_{m n}^{r s} a_{n}^{(s) \nu}+\sqrt{\alpha^{\prime}} \sum_{r, s} \sum_{n \geq 1} \eta_{\mu \nu} p^{(r) \mu} V_{0 n}^{r s} a_{n}^{(s) \nu}+\frac{\alpha^{\prime}}{2} \sum_{r} \eta_{\mu \nu} p^{(r) \mu} V_{00}^{r r} p^{(r) \nu} .
$$

Here $a_{m}^{\mu}$ are the bosonic oscillators normalized such that $\left[a_{m}^{\mu}, a_{n}^{\nu}\right]=\delta_{m,-n} \eta^{\mu \nu}$, and $p^{\mu}$ is the center of mass momentum. The Neumann coefficients $V_{m n}^{r s}$ were given in terms of coefficients of generating functions in [48,49]. They were diagonalized in [50]; the common eigenvectors $v_{n}(\kappa)$ for $M_{m n}^{r s}:=$ $C V_{m n}^{r s}$ with $1 \leq r, s \leq 3, C_{m n}=(-1)^{m} \delta_{m, n}$ are labeled by a continuous parameter $\kappa \in \mathbb{R}$, i. e.,

$$
M_{m n}^{r s} v_{n}(\kappa)=\mu^{r s}(\kappa) v_{m}(\kappa) .
$$

Here and in the following, a summation from 1 to $\infty$ over repeated indices is implied. The eigenvalues were found to be

$$
\begin{aligned}
& \mu^{11}(\kappa)=-\frac{1}{1+2 \cosh \frac{\pi \kappa}{2}}, \\
& \mu^{12}(\kappa)=\frac{1+\cosh \frac{\pi \kappa}{2}+\sinh \frac{\pi \kappa}{2}}{1+2 \cosh \frac{\pi \kappa}{2}}, \\
& \mu^{21}(\kappa)=\frac{1+\cosh \frac{\pi \kappa}{2}-\sinh \frac{\pi \kappa}{2}}{1+2 \cosh \frac{\pi \kappa}{2}} .
\end{aligned}
$$

Diagonalization of the fermionic coefficients. It was shown that solutions to the ghost part of the vacuum string field theory equations may be obtained from certain projectors of the twisted $b c$ system [23]. In this theory, the energy-momentum tensor of the untwisted $b c$ system for the critical bosonic string is supplemented by $-\partial J_{b c}$, the negative derivative of the ghost number current. This shifts the weights of $b$ to 1 and of $c$ to 0 , resulting in a first order system $b^{\prime} c^{\prime}$ of central charge $c_{b^{\prime} c^{\prime}}=-2$.

It turns out [36] that this first order system is exactly the fermionic part of string field theory for strings with $\mathrm{N}=2$ world-sheet supersymmetry. This theory possesses a twisted $\mathrm{N}=4$ superconformal invariance on the world-sheet and describes the dynamics of $\mathrm{N}=2$ strings in a (four-dimensional) Kähler space-time; therefore, two complex bosons $Z^{a}$ (with $a=1,2$ ) and their twisted superpartners $\psi^{+a}$ and $\psi^{-\bar{a}}$ are needed. For each $a$, it was shown in [36] that the fermionic $\psi^{+a} \psi^{-\bar{a}}$ system coincides with the twisted $b c$ system described above. ${ }^{4}$ For the following, it is thus irrelevant whether we are speaking of twisted $b c$ systems or of $\psi^{ \pm}$systems. For convenience, we mostly choose the latter alternative. As a trivial difference, however, it should be noted that we normalize the anticommutation relations for the $\psi^{ \pm}$system in such a way that they match complex fermions,

$$
\left\{\psi_{m}^{+a}, \psi_{n}^{-\bar{a}}\right\}=2 \eta^{a \bar{a}} \delta_{m,-n}
$$

for a Kähler metric with components $\eta^{a \bar{a}}$, whereas the normalization for the twisted $b c$ system conventionally differs from this by a factor of 2 .

\footnotetext{
${ }^{4}$ As indicated by the central charges, the reparametrization anomalies of one $\psi^{ \pm}$system and one $Z \bar{Z}$ system exactly cancel.
} 
As fields of integral weight, both $\psi^{+}$and $\psi^{-}$are integer-moded. In particular, the spin 0 field $\psi^{+}$has a zero-mode on the sphere. In analogy to the $b c$ system there are thus two vacua at the same energy level: the bosonic $S L(2, \mathbb{R})$-invariant vacuum $|0\rangle=:|\downarrow\rangle$ is annihilated by the Virasoro modes $L_{m \geq-1}$ and $\psi_{m>0}^{+}, \psi_{m \geq 0}^{-}$; its fermionic partner, $|\uparrow\rangle:=\psi_{0}^{+}|\downarrow\rangle$, is annihilated by $\psi_{m>0}^{+}, \psi_{m>0}^{-}$. To get nonvanishing fermionic correlation functions, we need one $\psi^{+}$-insertion, i.e., $\langle\downarrow \mid \downarrow\rangle=\langle\uparrow \mid \uparrow\rangle=0$, $\langle\downarrow \mid \uparrow\rangle=1$.

The interaction vertex for the fermionic $\psi^{ \pm}$system is then given by

$$
\left\langle V_{3}\right|=(\langle\uparrow \uparrow \downarrow|+\langle\uparrow \downarrow \uparrow|+\langle\downarrow \uparrow \uparrow|) \exp \left[\frac{1}{4} \sum_{r, s} \sum_{k=1, l=0}^{\infty} \psi_{k}^{+(r)} N_{k l}^{r s} \psi_{l}^{-(s)}\right]
$$

in terms of Neumann coefficients $N_{k l}^{r s}$ with $1 \leq r, s \leq 3$. For the nonzero-mode part of these coefficients it was shown in [36] (cf. also [37]) that they are related to the bosonic coefficients $N_{m n}^{r s}$ by

$$
N_{m n}^{r s}=2 \sqrt{\frac{m}{n}} V_{m n}^{r s}, \quad m, n \geq 1 .
$$

This renders the diagonalization almost trivial. Consider a matrix $U$ diagonalizing $V_{m n}^{r s}$ with $m, n \geq 1$ into a matrix $D$ by adjoint action. Then one can immediately diagonalize the nonzeromode part of $N_{m n}^{r s}$ as

$$
D_{k l}=U_{k m}^{-1} 2 V_{m n}^{r s} U_{n l}=U_{k m}^{-1}\left(\frac{1}{\sqrt{E}} N^{r s} \sqrt{E}\right)_{m n} U_{n l}=: U_{k m}^{\prime-1} N_{m n}^{r s} U_{n l}^{\prime},
$$

where we have introduced the matrix $E_{m n}=m \delta_{m n}$. Obviously, $V_{m n}^{r s}$ and $N_{m n}^{r s}$ share the same eigenvalues (2.4) (up to a factor of 2 ), ${ }^{5}$ and it follows that

$$
\begin{aligned}
& \left(\sqrt{E} 2 M^{r s} \frac{1}{\sqrt{E}}\right)_{m n}(\sqrt{E} \cdot v(\kappa))_{n}=2 \mu^{r s}(\kappa)(\sqrt{E} \cdot v(\kappa))_{m}, \\
& \left(v(\kappa) \cdot \frac{1}{\sqrt{E}}\right)_{m}\left(\sqrt{E} 2 M^{r s} \frac{1}{\sqrt{E}}\right)_{m n}=2 \mu^{r s}(\kappa)\left(v(\kappa) \cdot \frac{1}{\sqrt{E}}\right)_{n},
\end{aligned}
$$

which suggests the definitions

$$
v_{m}^{+}(\kappa):=(\sqrt{E} \cdot v(\kappa))_{m}=\sqrt{m} v_{m}(\kappa), \quad v_{m}^{-}(\kappa):=\left(\frac{1}{\sqrt{E}} \cdot v(\kappa)\right)_{m}=\frac{1}{\sqrt{m}} v_{m}(\kappa) .
$$

From [38] one derives the obvious relations

$$
\int_{-\infty}^{\infty} d \kappa v_{m}^{+}(\kappa) v_{n}^{-}(\kappa)=\delta_{m n}, \quad \sum_{m} v_{m}^{+}(\kappa) v_{m}^{-}\left(\kappa^{\prime}\right)=\delta\left(\kappa-\kappa^{\prime}\right),
$$

which can be split into even and odd parts to give

$$
\begin{aligned}
2 \int_{0}^{\infty} d \kappa v_{2 m}^{+}(\kappa) v_{2 n}^{-}(\kappa) & =\delta_{m n}, & 2 \int_{0}^{\infty} d \kappa v_{2 m+1}^{+}(\kappa) v_{2 n+1}^{-}(\kappa) & =\delta_{m n}, \\
2 \sum_{n=1}^{\infty} v_{2 n}^{+}(\kappa) v_{2 n}^{-}\left(\kappa^{\prime}\right) & =\delta\left(\kappa-\kappa^{\prime}\right), & 2 \sum_{n=1}^{\infty} v_{2 n-1}^{+}(\kappa) v_{2 n-1}^{-}\left(\kappa^{\prime}\right) & =\delta\left(\kappa-\kappa^{\prime}\right)
\end{aligned}
$$

\footnotetext{
${ }^{5}$ Since $N_{0 n}^{r s}=0$, it is obvious that the inclusion of the zero-mode part (i.e., admitting $m, n \geq 0$ ) simply adds an eigenvalue 0 to the spectrum.
} 
for $\kappa>0$. They are given by the generating functionals

$$
\begin{aligned}
& f_{v_{e}^{-}}(\kappa, z)=\sum_{n=1}^{\infty} v_{2 n}^{-}(\kappa) z^{2 n}=\frac{1}{\sqrt{\mathcal{N}(\kappa)} \kappa}(1-\cosh \kappa Z), \\
& f_{v_{o}^{-}}(\kappa, z)=\sum_{n=1}^{\infty} v_{2 n-1}^{-}(\kappa) z^{2 n-1}=\frac{1}{\sqrt{\mathcal{N}(\kappa)} \kappa} \sinh \kappa Z, \\
& f_{v_{e}^{+}}(\kappa, z)=\sum_{n=1}^{\infty} v_{2 n}^{+}(\kappa) z^{2 n}=-\frac{1}{\sqrt{\mathcal{N}(\kappa)}} \frac{z}{1+z^{2}} \sinh \kappa Z, \\
& f_{v_{o}^{+}}(\kappa, z)=\sum_{n=1}^{\infty} v_{2 n-1}^{-}(\kappa) z^{2 n-1}=\frac{1}{\sqrt{\mathcal{N}(\kappa)}} \frac{z}{1+z^{2}} \cosh \kappa Z
\end{aligned}
$$

with $Z:=\tan ^{-1} z$ and $\mathcal{N}(\kappa)=\frac{2}{\kappa} \sinh \frac{\pi \kappa}{2}$. We define the continuous modes

$$
\begin{aligned}
\psi_{e, \kappa}^{-\dagger}:=\sqrt{2} \sum_{n=1}^{\infty} v_{2 n}^{-}(\kappa) \psi_{-2 n}^{-}, & \psi_{o, \kappa}^{-\dagger}:=-\sqrt{2} \mathrm{i} \sum_{n=1}^{\infty} v_{2 n-1}^{-}(\kappa) \psi_{-2 n+1}^{-}, \\
\psi_{e, \kappa}^{+\dagger}:=\sqrt{2} \sum_{n=1}^{\infty} v_{2 n}^{+}(\kappa) \psi_{-2 n}^{+}, & \psi_{o, \kappa}^{+\dagger}:=-\sqrt{2} \mathrm{i} \sum_{n=1}^{\infty} v_{2 n-1}^{+}(\kappa) \psi_{-2 n+1}^{+}
\end{aligned}
$$

The factors of $\mathrm{i}$ are chosen in such a way that the BPZ conjugate of $\psi_{o, \kappa}^{+}\left(\psi_{o, \kappa}^{-}\right)$coincides with (minus) the hermitean conjugate; similar relations are true for the even components. They satisfy the anticommutation relations

$$
\left\{\psi_{e, \kappa}^{+\dagger}, \psi_{e, \kappa^{\prime}}^{-}\right\}=2 \delta\left(\kappa-\kappa^{\prime}\right), \quad\left\{\psi_{o, \kappa}^{+\dagger}, \psi_{o, \kappa^{\prime}}^{-}\right\}=2 \delta\left(\kappa-\kappa^{\prime}\right)
$$

following from the anticommutation relations of $\psi_{n}^{+}$and $\psi_{n}^{-}$and the completeness relations of the eigenvectors $v_{n}^{+}(\kappa)$ and $v_{n}^{-}(\kappa)$. The relations (2.14) can be inverted:

$$
\begin{array}{lll}
\psi_{-2 n}^{-}=\sqrt{2} \int_{0}^{\infty} d \kappa v_{2 n}^{+}(\kappa) \psi_{e, \kappa}^{-\dagger}, & \psi_{-2 n+1}^{-}=\sqrt{2} \mathrm{i} \int_{0}^{\infty} d \kappa v_{2 n-1}^{+}(\kappa) \psi_{o, \kappa}^{-\dagger}, \\
\psi_{-2 n}^{+}=\sqrt{2} \int_{0}^{\infty} d \kappa v_{2 n}^{-}(\kappa) \psi_{e, \kappa}^{+\dagger}, & \psi_{-2 n+1}^{+}=\sqrt{2} \mathrm{i} \int_{0}^{\infty} d \kappa v_{2 n-1}^{-}(\kappa) \psi_{o, \kappa}^{+\dagger} .
\end{array}
$$

We will use the continuous modes to rewrite the interaction vertex (2.6) in Moyal form.

The interaction vertex in the continuous basis. In this paper, we restrict to the nonzeromode part of the vertex. Only for these modes the connection to the bosonic vertex holds, and we find a correspondence of the projectors in both sectors. This is tantamount to the choice of a generalization of the Siegel gauge condition $\psi_{0}^{-}|\phi\rangle=0$. All known surface state projectors (which factorize into projectors for each value of $\kappa$ ) satisfy this condition; they are based on the $|\downarrow\rangle$-vacuum.

The nonzero-mode part of the ket-interaction vertex reads

$$
\left|V_{3}\right\rangle^{\prime}=\exp \left[-\frac{1}{4} \sum_{r, s} \sum_{k, l \geq 1} \psi_{-k}^{+(r)}(C N C)_{k l}^{r s} \psi_{-l}^{-(s)}\right]|\Omega\rangle .
$$

Using the relations of the previous paragraph we can rewrite it as

$$
\begin{aligned}
\left|V_{3}\right\rangle^{\prime} & =\exp \left[-\frac{1}{2} \sum_{r, s} \sum_{k, l \geq 1} \psi_{-k}^{+(r)}\left(C \sqrt{E} V^{r s} \frac{1}{\sqrt{E}} C\right)_{k l} \psi_{-l}^{-(s)}\right]|\Omega\rangle \\
& =\exp \left[-\frac{1}{2} \sum_{r, s} \sum_{k, l \geq 1} \psi_{-k}^{+(r)}\left(\sqrt{E} M^{r s} C \frac{1}{\sqrt{E}}\right)_{k l} \psi_{-l}^{-(s)}\right]|\Omega\rangle .
\end{aligned}
$$


Splitting in even and odd parts and inserting the continuous basis (2.14) we obtain

$$
\begin{aligned}
\sum_{r, s} \sum_{k, l \geq 1} \psi_{-k}^{+(r)} & \left(\sqrt{E} M^{r s} C \frac{1}{\sqrt{E}}\right)_{k l} \psi_{-l}^{-(s)} \\
=\sum_{r, s} \frac{1}{2} \int_{0}^{\infty} d \kappa\left[\left(\mu^{r s}(\kappa)+\mu^{s r}(\kappa)\right)\left(\psi_{e, \kappa}^{+(r)} \psi_{e, \kappa}^{-(s)^{\dagger}}+\psi_{o, \kappa}^{+(r)^{\dagger}} \psi_{o, \kappa}^{-(s)^{\dagger}}\right)\right. & \\
& \left.\left.-\left(\mu^{r s}(\kappa)-\mu^{s r}(\kappa)\right)\left(\mathrm{i} \psi_{e, \kappa}^{+(r)^{\dagger}} \psi_{o, \kappa}^{-(s)^{\dagger}}-\mathrm{i} \psi_{o, \kappa}^{+(r)^{\dagger}} \psi_{e, \kappa}^{-(s)}\right)^{\dagger}\right)\right] .
\end{aligned}
$$

For notational ease we introduce

$$
\vec{\psi}_{\kappa}^{ \pm \dagger}=\left(\begin{array}{c}
\psi_{e, \kappa}^{ \pm \dagger} \\
\psi_{o, \kappa}^{ \pm} \dagger
\end{array}\right)
$$

in this vector notation, the nonzero-mode part of the interaction vertex eventually takes the form

$$
\left|V_{3}\right\rangle^{\prime}=\exp \left[-\frac{1}{2} \sum_{r, s} \int_{0}^{\infty} d \kappa\left(\mu^{(r s)}(\kappa) \vec{\psi}_{\kappa}^{+\dagger(r)} \cdot \vec{\psi}_{\kappa}^{-\dagger(s)}+\mu^{[r s]}(\kappa) \vec{\psi}_{\kappa}^{+\dagger(r)} \cdot \sigma_{y} \cdot \vec{\psi}_{\kappa}^{-\dagger(s)}\right)\right]|\Omega\rangle,
$$

where $\sigma_{y}$ denotes the second Pauli matrix, and $\mu^{(r s)}(\kappa)$ and $\mu^{[r s]}(\kappa)$ are the symmetric and antisymmetric parts of $\mu^{r s}(\kappa)$, respectively.

Identification of Moyal structures. We can now readily transform the nonzero-mode part of the interaction vertex (2.21) into a form which resembles the structure of the oscillator vertex for the Moyal-Weyl product $[38,41]$ for anticommuting quantities. As a first step, observe that $(2.21)$ can be rewritten as

$$
\left|V_{3}\right\rangle^{\prime}=\exp \left[-\frac{1}{2} \sum_{r, s} \int_{0}^{\infty} d \kappa \vec{\psi}_{\kappa}^{+(r) \dagger} \cdot \widetilde{V}^{r s} \cdot \vec{\psi}_{\kappa}^{-(s) \dagger}\right]|\Omega\rangle
$$

where $\widetilde{V}^{r s}$ is the $6 \times 6$ matrix

$$
\tilde{V}^{r s}=\frac{1}{\theta^{2}(\kappa)+12}\left(\begin{array}{ccc}
\theta^{2}(\kappa)-4 & 8+4 \theta(\kappa) \sigma_{y} & 8-4 \theta(\kappa) \sigma_{y} \\
8-4 \theta(\kappa) \sigma_{y} & \theta^{2}(\kappa)-4 & 8+4 \theta(\kappa) \sigma_{y} \\
8+4 \theta(\kappa) \sigma_{y} & 8-4 \theta(\kappa) \sigma_{y} & \theta^{2}(\kappa)-4
\end{array}\right)^{r s}
$$

and $\theta(\kappa)=2 \tanh \frac{\pi \kappa}{4}$ is the unique solution to the equations

$$
\begin{aligned}
\mu^{11}(\kappa) & =\frac{\theta^{2}(\kappa)-4}{\theta^{2}(\kappa)+12}, \\
\frac{1}{2}\left(\mu^{12}(\kappa)+\mu^{21}(\kappa)\right) & =\frac{8}{\theta^{2}(\kappa)+12} \\
\frac{1}{2}\left(\mu^{12}(\kappa)-\mu^{21}(\kappa)\right) & =\frac{4 \theta(\kappa)}{\theta^{2}(\kappa)+12} .
\end{aligned}
$$

As a second step, we contract the interaction vertex with eigenvectors of the position operators $\vec{x}_{\kappa}=\left(\begin{array}{c}x_{e, \kappa} \\ x_{o, \kappa}\end{array}\right)=\frac{\mathrm{i}}{\sqrt{2}}\left(\vec{\psi}_{\kappa}^{-}-\vec{\psi}_{\kappa}^{-\dagger}\right)$ and $\vec{y}_{\kappa}=\left(\begin{array}{c}y_{e, \kappa} \\ y_{o}, \kappa\end{array}\right)=\frac{1}{\sqrt{2}}\left(\vec{\psi}_{\kappa}^{+}+\vec{\psi}_{\kappa}^{+\dagger}\right)$ in order to obtain an integral kernel representation for the Moyal product [41]. The eigenvectors are given by

$$
\begin{aligned}
\langle\vec{X}|:=\langle\vec{x}, \vec{y}|=\langle\downarrow| \exp \left[-\frac{1}{2} \int_{0}^{\infty} d \kappa\left(\vec{\psi}_{\kappa}^{+} \cdot \vec{\psi}_{\kappa}^{-}+\mathrm{i} \sqrt{2} \vec{\psi}_{\kappa}^{+} \cdot \vec{x}(\kappa)\right.\right. \\
\left.\left.-\sqrt{2} \vec{y}(\kappa) \cdot \vec{\psi}_{\kappa}^{-}+\mathrm{i} \vec{x}(\kappa) \cdot \vec{y}(\kappa)\right)\right] .
\end{aligned}
$$


Then the integral kernel for the star product is given by

$$
\begin{aligned}
& K\left(\vec{X}^{(1)}, \vec{X}^{(2)} ; \vec{X}^{(3)}\right)=\left\langle\vec{X}^{(3)}\right|\left\langle\vec{X}^{(1)}\right|\left\langle\vec{X}^{(2)} \mid V_{3}\right\rangle \\
& \quad=\exp \left[\delta(0) \int d \kappa \ln \left(\operatorname{det}\left(1+\widetilde{V}^{r s}\right)\right)\right] \exp \left[-\mathrm{i} \sum_{r, s} \int_{0}^{\infty} d \kappa \vec{x}^{(r)}(\kappa) \cdot W^{r s} \cdot \vec{y}^{(s)}(\kappa)\right],
\end{aligned}
$$

where $W^{r s}:=\frac{1-\widetilde{V}^{r s}}{1+\widetilde{V}^{r s}}$ takes the form

$$
W^{r s}=\theta^{-1}\left(\begin{array}{ccc}
0 & -\sigma_{y} & \sigma_{y} \\
\sigma_{y} & 0 & -\sigma_{y} \\
-\sigma_{y} & \sigma_{y} & 0
\end{array}\right)^{r s},
$$

and $\operatorname{det}\left(1+\tilde{V}^{r s}\right)=\frac{64 \theta^{4}(\kappa)}{\left(\theta^{2}(\kappa)+12\right)^{2}}$. This is a product over Moyal kernels for a tensor product of two Clifford algebras [41] for each $\kappa>0$ since

$$
-\mathrm{i} \vec{x}^{(r)}(\kappa) W^{r s} \vec{y}^{(s)}(\kappa)=x_{e}^{(r)}(\kappa) K^{r s} y_{o}^{(s)}(\kappa)-x_{o}^{(r)}(\kappa) K^{r s} y_{e}^{(s)}(\kappa),
$$

where

$$
K^{r s}=\theta^{-1}\left(\begin{array}{ccc}
0 & 1 & -1 \\
-1 & 0 & 1 \\
1 & -1 & 0
\end{array}\right) .
$$

From this, we read off that for a canonically normalized star product we can identify two separate continuous Moyal-Weyl pairs, namely $\left(x_{e}(\kappa), y_{o}(\kappa)\right)$ and $\left(x_{o}(\kappa), y_{e}(\kappa)\right)$ with

$$
\begin{aligned}
& \left\{x_{e}(\kappa) \stackrel{\star}{,} y_{o}\left(\kappa^{\prime}\right)\right\}=2 \theta(\kappa) \delta\left(\kappa-\kappa^{\prime}\right), \\
& \left\{x_{o}(\kappa) \stackrel{\star}{,} y_{e}\left(\kappa^{\prime}\right)\right\}=-2 \theta(\kappa) \delta\left(\kappa-\kappa^{\prime}\right),
\end{aligned}
$$

which is twice the noncommutativity for one real boson. All other anticommutators vanish. ${ }^{6}$ This result can be checked if one uses the path integral measure for which the norm of the ground state

$$
\Psi_{|0\rangle}=\exp \left[-\frac{\mathrm{i}}{2} \int_{0}^{\infty} d \kappa(\vec{x}(\kappa) \cdot \vec{y}(\kappa))\right]
$$

(which is recovered from contraction of (2.25) with the vacuum) is one.

Cancellation of determinants. It is worthwhile to pause here for a moment and have a closer look at the meaning of the result in eq. (2.26) for $\mathrm{N}=2$ string field theory. As could have been expected the Moyal kernel differs from the result obtained in [41] essentially by the normalization factor. For $D / 2 \psi^{ \pm}$-pairs, i. e., in a spacetime of real even dimension $D$, the normalization factor can be read off from (2.26). The analog of the constant $C^{\prime}$ of [38] evaluates to

$$
\mathcal{N}_{\psi}=\exp \left[-\delta(0) \frac{D}{2} \int d \kappa \ln \left(\frac{\left(\theta^{2}(\kappa)+12\right)^{2}}{64}\right)\right]
$$

In [38] a cancellation of such normalization factors between the matter and ghost sector was advocated. It turned out [41] that this cancellation does not occur in bosonic string field theory, at least for the reduced star product of the ghost sector. Here the situation is quite different. The normalization factor for the bosonic (matter) part of the star product computed in [38] is proportional to the number of spacetime dimensions $D$ and reads

$$
\mathcal{N}_{X}=\exp \left[\delta(0) D \int d \kappa \ln \left(\frac{1}{8}\left(\theta^{2}(\kappa)+12\right)\right)\right]
$$

\footnotetext{
${ }^{6}$ For $\kappa=0$, the algebra is anticommutative.
} 
Hence, taking into account that due to eqs. (2.10) and (2.11) the eigenvalue densities ${ }^{7}$ for bosons and fermions are equal

$$
\rho(\kappa)_{X}=\sum_{n=1}^{\infty} v_{n}(\kappa) v_{n}(\kappa)=\sum_{n=1}^{\infty} v_{n}^{-}(\kappa) v_{n}^{+}(\kappa)=\rho(\kappa)_{\psi},
$$

the advocated cancellation

$$
\mathcal{N}_{X} \mathcal{N}_{\psi}=1
$$

indeed happens in any even dimension. ${ }^{8}$ This is a distinguished feature of $\mathrm{N}=2$ string field theory. A similar cancellation has been observed for the anomaly of midpoint preserving reparametrizations in [36]. Both cancellations are consequences of relation (2.7).

The same is true for the canonical normalizations, which are given by

$$
\mathcal{N}_{\psi, \text { can. }}=\exp \left[\delta(0) \frac{D}{2} \int d \kappa \ln \left(\theta^{4}\right)\right]
$$

for the fermions and by

$$
\mathcal{N}_{X, \text { can. }}=\exp \left[-\delta(0) D \int d \kappa \ln \left(\theta^{2}\right)\right]
$$

for the bosons, where the path integral measure is normalized such that the norm of the bosonic as well as the norm of the fermionic ground state is 1 . The full Witten star product of $\mathrm{N}=2$ string field theory is a canonically normalized continuous tensor product of Moyal-Weyl products.

\section{$3 \quad$ Star algebra projectors}

As known from noncommutative field theories, the computation of star products can be facilitated considerably by making use of the Moyal-Weyl map. In our case, we can do the same; now, string fields are translated into operators in some auxiliary Fock space $\mathcal{H}_{\text {aux }}$, and star products are computed via pairs of (Grassmann-odd) pairs of creation and annihilation operators for each $\kappa$. This makes it possible to identify a large subclass of projectors of the twisted ghost part of the star algebra, namely those which are projectors for each $\kappa>0$ separately.

Operator representation of the star product. The anticommutation relations (2.30) suggest that the Moyal-Weyl map should have the following properties:

$$
\begin{array}{ll}
\frac{x_{e}(\kappa)}{\sqrt{2 \theta(\kappa)}} \mapsto c_{\kappa}, & \frac{y_{o}(\kappa)}{\sqrt{2 \theta(\kappa)}} \mapsto c_{\kappa}^{\dagger}, \\
\frac{\mathrm{i} x_{o}(\kappa)}{\sqrt{2 \theta(\kappa)}} \mapsto d_{\kappa}, & \frac{\mathrm{i} y_{e}(\kappa)}{\sqrt{2 \theta(\kappa)}} \mapsto d_{\kappa}^{\dagger},
\end{array}
$$

where the operators act in an auxiliary Fock space with vacua

$$
c_{\kappa}|0\rangle_{c}=0, \quad d_{\kappa}|0\rangle_{d}=0 .
$$

In the following we will investigate the star product for a single $\kappa$. The Moyal-Weyl map assigns to each functional $\Phi\left(x_{e}(\kappa), x_{o}(\kappa), y_{e}(\kappa), y_{o}(\kappa)\right)$ an operator $\hat{\Phi}\left(c_{\kappa}, d_{\kappa}, d_{\kappa}^{\dagger}, c_{\kappa}^{\dagger}\right)$ acting in the auxiliary

\footnotetext{
${ }^{7}$ For a discussion of the spectral density see [51, 52].

${ }^{8}$ Note that this will also hold for the finite part of the spectral density after regularization.
} 
Fock space. In principle, this assignment involves a particular ordering prescription for the operators. We decide to keep the ordering which is inherited from the expressions as string fields. It is understood that under the Moyal-Weyl map,

$$
\Phi \star \Psi \mapsto \hat{\Phi} \hat{\Psi} .
$$

In this way, the star anticommutators (2.30) are mapped to anticommutators of operators. In order to be able to obtain reasonable results in the continuum normalization, we have to regularize the delta distributions in (2.30). To this aim, we introduce a level regulator $L$ as in $[50,53,54]$ and formally set $\delta(0) \sim \frac{\log L}{2 \pi}=: R$. Under the Moyal-Weyl map, the regularized star anticommutators can be rewritten as

$$
\left\{c_{\kappa}, c_{\kappa}^{\dagger}\right\}=R, \quad\left\{d_{\kappa}, d_{\kappa}^{\dagger}\right\}=R,
$$

with all other anticommutators vanishing.

Projectors from the Moyal-Weyl map. We will now take advantage of the map defined above to classify projectors for the combined system of fermionic operators $c_{\kappa}, c_{\kappa}^{\dagger}$ and $d_{\kappa}$, $d_{\kappa}^{\dagger}$. It will turn out that the combined system allows for a much larger variety of projectors as compared to a single set of operators. Namely, for a bosonic rank one projector built out of, say, $c_{\kappa}$ and $c_{\kappa}^{\dagger}$, one finds that the only possibilities are $\mathbb{1}, \frac{1}{R} c_{\kappa} c_{\kappa}^{\dagger}$ and $\frac{1}{R} c_{\kappa}^{\dagger} c_{\kappa}$. These operators correspond to $|0\rangle_{c c}\langle 0|+| 1\rangle_{c c}\langle 1|,| 0\rangle_{c c}\langle 0|$ and $|1\rangle_{c c}\langle 1|$, respectively, where $|1\rangle_{c}=c_{\kappa}^{\dagger}|0\rangle_{c}$. Of course, the combined system of oscillators will contain more projectors than the naive ones build as products of projectors in each $\kappa$-subsystem; but, as mentioned above, this paper is devoted to an investigation of states which are projectors for each value of $\kappa$.

We insert the ansatz

$$
\hat{P}_{\kappa}=\alpha \mathbb{1}+\beta c_{\kappa} c_{\kappa}^{\dagger}+\gamma d_{\kappa} d_{\kappa}^{\dagger}+\delta c_{\kappa} d_{\kappa}+\varepsilon c_{\kappa}^{\dagger} d_{\kappa}^{\dagger}+\varphi c_{\kappa} d_{\kappa}^{\dagger}+\eta c_{\kappa}^{\dagger} d_{\kappa}+\lambda c_{\kappa} c_{\kappa}^{\dagger} d_{\kappa} d_{\kappa}^{\dagger}
$$

into the projector condition $\hat{P}_{\kappa} \hat{P}_{\kappa}=\hat{P}_{\kappa}$ and read off equations for the coefficient functions. This yields the following set of equations:

$$
\begin{aligned}
\alpha & =\alpha^{2}-R^{2} \varepsilon \delta, \\
\beta & =2 \alpha \beta+R \beta^{2}+R \varepsilon \delta-R \varphi \eta, \\
\gamma & =2 \alpha \gamma+R \gamma^{2}+R \varepsilon \delta-R \varphi \eta, \\
\delta & =\left(2 \alpha+R \beta+R \gamma+R^{2} \lambda\right) \delta, \\
\varepsilon & =\left(2 \alpha+R \beta+R \gamma+R^{2} \lambda\right) \varepsilon, \\
\varphi & =(2 \alpha+R \beta+R \gamma) \varphi, \\
\eta & =(2 \alpha+R \beta+R \gamma) \eta, \\
\lambda & =R^{2} \lambda^{2}+2(\alpha+R \beta+R \gamma) \lambda+2 \beta \gamma-2 \varepsilon \delta+2 \varphi \eta .
\end{aligned}
$$

Subtracting eq. (3.6c) from eq. (3.6b) one finds

$$
(\beta-\gamma)(2 \alpha-1+R \beta+R \gamma)=0 .
$$

We will use this condition to classify the solutions to (3.6):

(I): $2 \alpha+R \beta+R \gamma=1$

(a): $\delta \neq 0 \vee \varepsilon \neq 0 \Rightarrow \lambda=0, \delta \varepsilon=\beta \gamma+\varphi \eta=\frac{1}{R^{2}}\left(\alpha^{2}-\alpha\right)$. 
(b): $\delta=\varepsilon=0 \Rightarrow \alpha \in\{0,1\}$.

(b1): $\alpha=0 \Rightarrow \beta=\frac{1}{R}-\gamma, \gamma\left(\gamma-\frac{1}{R}\right)=\varphi \eta, \lambda \in\left\{0,-\frac{1}{R^{2}}\right\}$.

(b2): $\alpha=1 \Rightarrow \beta=-\frac{1}{R}-\gamma, \gamma\left(\gamma+\frac{1}{R}\right)=\varphi \eta, \lambda \in\left\{0, \frac{1}{R^{2}}\right\}$.

(II): $\beta=\gamma$

(a): $\varphi \neq 0 \vee \eta \neq 0 \Rightarrow \alpha=\frac{1}{2}-R \gamma, \delta \varepsilon=\gamma^{2}-\frac{1}{4 R^{2}}, \varphi \eta=-\frac{1}{4 R^{2}}$.

(a1): $\delta \varepsilon \neq 0 \Rightarrow \lambda=0$.

$(\mathrm{a} 2):(\delta \neq 0, \varepsilon=0) \vee(\delta=0, \varepsilon \neq 0) \Rightarrow \lambda=0,\left(\gamma=\frac{1}{2 R}, \alpha=0\right) \vee\left(\gamma=-\frac{1}{2 R}, \alpha=1\right)$.

(a3): $\delta=\varepsilon=0 \Rightarrow\left(\gamma=\frac{1}{2 R}, \alpha=0, \lambda \in\left\{0,-\frac{1}{R^{2}}\right\}\right) \vee\left(\gamma=-\frac{1}{2 R}, \alpha=1, \lambda \in\left\{0, \frac{1}{R^{2}}\right\}\right)$.

(b): $\varphi=\eta=0 \Rightarrow \gamma=\frac{1-\alpha}{R} \vee \gamma=-\frac{\alpha}{R}, \lambda=\frac{1-2 \alpha}{R^{2}}-\frac{2 \gamma}{R} \vee \lambda=-\frac{2 \gamma}{R}, \delta \varepsilon=\frac{\alpha^{2}-\alpha}{R^{2}}$.

(b1): $\delta \varepsilon \neq 0$.

(b2): $(\delta \neq 0, \varepsilon=0) \vee(\delta=0, \varepsilon \neq 0) \Rightarrow$

$(\alpha, \gamma, \lambda) \in\left\{\left(0, \frac{1}{R},-\frac{1}{R^{2}}\right),\left(0,0, \frac{1}{R^{2}}\right),\left(1,0,-\frac{1}{R^{2}}\right),\left(1,-\frac{1}{R}, \frac{1}{R^{2}}\right)\right\}$.

(b3): $\delta=\varepsilon=0 \Rightarrow(\alpha, \gamma, \lambda) \in\left\{\left(0, \frac{1}{R},-\frac{1}{R^{2}}\right),\left(0, \frac{1}{R},-\frac{2}{R^{2}}\right),\left(0,0, \frac{1}{R^{2}}\right),(0,0,0)\right.$,

$\left.\left(1,0,-\frac{1}{R^{2}}\right),(1,0,0),\left(1,-\frac{1}{R}, \frac{1}{R^{2}}\right),\left(1,-\frac{1}{R}, \frac{2}{R^{2}}\right)\right\}$.

The first and second level (denoted by roman numbers and latin letters) specify general conditions which have to be fulfilled simultaneously. If possible, the third level (denoted by an arabic number) lists all solutions divided into subclasses. Note that all variables not further specified in the third level still have to satisfy the conditions stated in the first and second level.

All of these solutions to the projector condition are valid for any $\kappa>0$. By taking the continuous tensor product one has to choose a different set of coefficients $(\alpha, \ldots, \lambda)$, i. e., the coefficients are promoted to functions of $\kappa$. Since the change of basis from discrete to continuous oscillators involves integrations it seems to be necessary to demand integrability of the coefficient functions. Later on, we will demand additional restrictions for certain subclasses of projectors. Apart from that, further restrictions would amount to specify the class of allowed string fields [19]. We will not impose more restrictions now, but instead leave this subject open for further work. ${ }^{9}$

Representation as states. In order to elucidate which states actually are parametrized by the projectors in the last paragraph, we first translate the ansatz (3.5) into string fields. The corresponding states will then be extracted, which, with the coefficients as in the above classification, are projector states.

The inverse Moyal-Weyl map determines the string field (localized at one fixed value of $\kappa$ )

$$
\begin{aligned}
P[\vec{x}(\kappa), \vec{y}(\kappa)]= & \alpha+\frac{(\beta+\gamma) R}{2}+\frac{\lambda R^{2}}{4}+\frac{2 \beta+\lambda R}{4 \theta} x_{e} y_{o}-\frac{2 \gamma+\lambda R}{4 \theta} x_{o} y_{e} \\
& +\frac{\mathrm{i} \delta}{2 \theta} x_{e} x_{o}-\frac{\mathrm{i} \varepsilon}{2 \theta} y_{e} y_{o}+\frac{\mathrm{i} \varphi}{2 \theta} x_{e} y_{e}-\frac{\mathrm{i} \eta}{2 \theta} x_{o} y_{o}-\frac{\lambda}{4 \theta^{2}} x_{e} y_{o} x_{o} y_{e}
\end{aligned}
$$

corresponding to the ansatz (3.5) if one makes use of the fact that, e.g., $x_{e} \star y_{o}=x_{e} y_{o}+\theta R$. Here, all star products are already evaluated in terms of the ordinary Grassmann product. The orthogonality of the sectors of the star algebra with different $\kappa$ allows us to restrict ourselves to one $\kappa$ for the time being.

In order to translate the string field (3.8) into a linear combination of Fock space states, we introduce the generating state

$$
|G\rangle=\exp \left[-\frac{1}{2 R} \sum_{\kappa}\left(\vec{\psi}_{\kappa}^{+\dagger} \cdot \vec{\psi}_{\kappa}^{-\dagger}+\sqrt{2} \mathrm{i} \vec{\lambda}(\kappa) \cdot \vec{\psi}_{\kappa}^{-\dagger}+\sqrt{2} \vec{\psi}_{\kappa}^{+\dagger} \cdot \vec{\mu}(\kappa)+\mathrm{i} \vec{\lambda}(\kappa) \cdot \vec{\mu}(\kappa)\right)\right]|\downarrow\rangle,
$$

\footnotetext{
${ }^{9}$ In principle, one can even try to employ different types of projectors for different $\kappa$ 's.
} 
which can be contracted with (the discretized version of) eq. (2.25) to give

$$
\langle\vec{X} \mid G\rangle \propto \exp \left(\frac{1}{2 R} \sum_{\kappa}(\vec{\lambda}(\kappa) \cdot \vec{x}(\kappa)-\vec{y}(\kappa) \cdot \vec{\mu}(\kappa))\right)
$$

up to a proportionality constant independent of $R, \kappa$. Neglecting this constant, $x_{o} y_{o}$, e.g., for a single $\kappa$ corresponds to $2 \mathrm{i} R \exp \left(-\frac{1}{2 R}\left(\psi_{\kappa, e}^{+\dagger} \psi_{\kappa, e}^{-\dagger}-\psi_{\kappa, o}^{+\dagger} \psi_{\kappa, o}^{-\dagger}\right)\right)|\downarrow\rangle$. Thus, the state corresponding to $(3.8)$ reads

$$
\begin{aligned}
\left|P_{\kappa}\right\rangle= & \left(f(\alpha, \beta, \gamma, \lambda)-\frac{\lambda R^{2}}{\theta^{2}}-\frac{\varphi R}{\theta}+\frac{\eta R}{\theta}\right)|\downarrow\rangle-\frac{1}{2 R}\left(f(\alpha, \beta, \gamma, \lambda)+\frac{\lambda R^{2}}{\theta^{2}}+\frac{\varphi R}{\theta}+\frac{\eta R}{\theta}\right) \psi_{\kappa, e}^{+\dagger} \psi_{\kappa, e}^{-\dagger}|\downarrow\rangle \\
& -\frac{1}{2 R}\left(f(\alpha, \beta, \gamma, \lambda)+\frac{\lambda R^{2}}{\theta^{2}}-\frac{\varphi R}{\theta}-\frac{\eta R}{\theta}\right) \psi_{\kappa, o}^{+\dagger} \psi_{\kappa, o}^{-\dagger}|\downarrow\rangle+\frac{2 \beta+\lambda R}{2 \theta} \mathrm{i} \psi_{\kappa, o}^{+\dagger} \psi_{\kappa, e}^{-\dagger}|\downarrow\rangle-\frac{2 \gamma+\lambda R}{2 \theta} \mathrm{i} \psi_{\kappa, e}^{+\dagger} \psi_{\kappa, o}^{-\dagger}|\downarrow\rangle \\
& -\frac{\mathrm{i} \delta}{\theta} \psi_{\kappa, e}^{-\dagger} \psi_{\kappa, o}^{-\dagger}|\downarrow\rangle-\frac{\mathrm{i} \varepsilon}{\theta} \psi_{\kappa, e}^{+\dagger} \psi_{\kappa, o}^{+\dagger}|\downarrow\rangle+\frac{1}{4 R^{2}}\left(f(\alpha, \beta, \gamma, \lambda)-\frac{\lambda R^{2}}{\theta^{2}}+\frac{\varphi R}{\theta}-\frac{\eta R}{\theta}\right) \psi_{\kappa, e}^{+\dagger} \psi_{\kappa, e}^{-\dagger} \psi_{\kappa, o}^{+\dagger} \psi_{\kappa, o}^{-\dagger}|\downarrow\rangle
\end{aligned}
$$

with

$$
f(\alpha, \beta, \gamma, \lambda)=\alpha+\frac{(\beta+\gamma) R}{2}+\frac{\lambda R^{2}}{4} .
$$

It is remarkable that our classification admits projectors which are not neutral and not even homogeneous in their $U(1)$ charge (those with $\delta \neq 0$ or $\varepsilon \neq 0$ ).

Reality condition. Admissible string fields are subject to a reality condition with respect to star conjugation, cf. [6,55]: The hermitean conjugate has to equal the BPZ conjugate of a given state. In our case (3.11) this leads to the condition that $\delta, \varepsilon, \varphi, \eta$, and $\lambda$ are real, as well as the linear combination $f(\alpha, \beta, \gamma, \lambda)$. Furthermore, the real parts of $\beta$ and $\gamma$ are restricted to be

$$
\operatorname{Re} \beta=\operatorname{Re} \gamma=-\frac{\lambda R}{2} .
$$

Imposing the reality condition in addition to eqs. (3.6) reduces the number of solutions in all classes:

(''): $2 \alpha+R \beta+R \gamma=1$

$\left(\mathrm{a}^{\prime}\right): \delta \neq 0 \vee \varepsilon \neq 0 \Rightarrow \lambda=0, \delta \varepsilon=\beta \gamma+\varphi \eta=\frac{1}{R^{2}}\left(\alpha^{2}-\alpha\right), \operatorname{Re} \beta=\operatorname{Re} \gamma=0$.

(b'): empty.

$\left(\mathrm{II}^{\prime}\right): \beta=\gamma$

$\left(\mathrm{a}^{\prime}\right): \varphi \neq 0 \vee \eta \neq 0 \Rightarrow \alpha=\frac{1}{2}-R \gamma, \delta \varepsilon=\gamma^{2}-\frac{1}{4 R^{2}}, \varphi \eta=-\frac{1}{4 R^{2}}$.

$\left(\mathrm{a} 1^{\prime}\right): \delta \varepsilon \neq 0 \Rightarrow \lambda=0, \operatorname{Re} \beta=\operatorname{Re} \gamma=0$.

$\left(\mathrm{a} 2^{\prime}\right):$ empty.

(a3'): $\delta=\varepsilon=0 \Rightarrow(\alpha, \gamma, \lambda) \in\left\{\left(0, \frac{1}{2 R},-\frac{1}{R^{2}}\right),\left(1,-\frac{1}{2 R}, \frac{1}{R^{2}}\right)\right\}$.

$\left(\mathrm{b}^{\prime}\right): \varphi=\eta=0 \Rightarrow \gamma=\frac{1-\alpha}{R} \vee \gamma=-\frac{\alpha}{R},\left(\lambda=\frac{1-2 \alpha}{R^{2}}-\frac{2 \gamma}{R}, \operatorname{Re} \alpha=\frac{1}{2}\right) \vee\left(\lambda=-\frac{2 \gamma}{R}, \gamma \in \mathbb{R}\right)$, $\delta \varepsilon=\frac{\alpha^{2}-\alpha}{R^{2}}$.

$\left(\mathrm{b} 1^{\prime}\right): \delta \varepsilon \neq 0$.

(b2'): empty.

(b3'): $\delta=\varepsilon=0 \Rightarrow(\alpha, \gamma, \lambda) \in\left\{\left(0, \frac{1}{R},-\frac{2}{R^{2}}\right),(0,0,0),(1,0,0),\left(1,-\frac{1}{R}, \frac{2}{R^{2}}\right)\right\}$.

From now on, we will switch to bra states, which makes the identification of surface states somewhat easier. 


\section{Squeezed state projectors}

In this section, we restrict to the subclass of squeezed state projectors which are BPZ-real and diagonal in the $\kappa$-basis. A general squeezed state in the fermionic sector has the form

$$
\langle S|=\langle\downarrow| \exp \left[\frac{1}{2} \sum_{m, n}\left(\psi_{m}^{+} S_{m n} \psi_{n}^{-}+\psi_{m}^{+} P_{m n} \psi_{n}^{+}+\psi_{m}^{-} M_{m n} \psi_{m}^{-}\right)\right],
$$

where we admit also projectors with indefinite $U(1)$ charge. We will see below that such projectors are included in our classification. Furthermore, we neglect possible normalization factors.

Squeezed state projectors in the diagonal basis. In the continuous basis, the squeezed states feature two $\kappa$-integrations in the exponent. Note, however, that we restricted our classification to projectors which are already projectors for each $\kappa$. Exactly if this is the case, $S_{m n},(E M)_{m n}$, $\left(E^{-1} P\right)_{m n}$, and the Neumann coefficients $N_{m n}^{r s}$ are simultaneously diagonalizable; ${ }^{10}$ and the exponent can be reduced to contain only one $\kappa$-integration. Namely, for $S_{m n}$, the twist properties of $v_{n}^{+}(\kappa)$

$$
v_{2 n}^{+}(-\kappa)=-v_{2 n}^{+}(\kappa), \quad v_{2 n+1}^{+}(-\kappa)=v_{2 n+1}^{+}(\kappa),
$$

imply that the even and odd parts are also eigenvectors of $S_{m n}$,

$$
\begin{aligned}
\sum_{n} S_{2 m, 2 n} v_{2 n}^{+}(\kappa) & =S_{e e}(\kappa) v_{2 m}^{+}(\kappa), & \sum_{n} S_{2 m+1,2 n} v_{2 n}^{+}(\kappa) & =S_{o e}(\kappa) v_{2 m+1}^{+}(\kappa), \\
\sum_{n} S_{2 m, 2 n+1} v_{2 n+1}^{+}(\kappa) & =S_{e o}(\kappa) v_{2 m}^{+}(\kappa), & \sum_{n} S_{2 m+1,2 n+1} v_{2 n+1}^{+}(\kappa) & =S_{o o}(\kappa) v_{2 m+1}^{+}(\kappa) .
\end{aligned}
$$

Here, $S_{e e}, S_{o e}, S_{e o}$, and $S_{o o}$, denote the corresponding eigenvalues. ${ }^{11}$ Thus, eq. (2.12) guarantees that

$$
\sum_{m, n} v_{2 m}^{-}\left(\kappa^{\prime}\right) S_{2 m, 2 n} v_{2 n}^{+}(\kappa)=\frac{1}{2} S_{e e}(\kappa) \delta\left(\kappa-\kappa^{\prime}\right)
$$

and similar relations hold for the other components. If one rewrites $\psi_{m}^{+} S_{m n} \psi_{n}^{-}$in terms of the continuously moded operators, the delta distributions on the right-hand side of eq. (4.4) can be used to remove the $\kappa^{\prime}$-integration. In the case of $P_{m n}$, we have

$$
\begin{aligned}
& \sum_{n}\left(E^{-1} P\right)_{2 m+1,2 n} v_{2 n}^{-}(\kappa)=p_{o e}(\kappa) v_{2 m+1}^{-}(\kappa) \\
& \Longrightarrow \sum_{m, n}\left(v^{-}\left(\kappa^{\prime}\right) E\right)_{2 m+1}\left(E^{-1} P\right)_{2 m+1,2 n} v_{2 n}^{-}(\kappa)=\frac{1}{2} p_{o e}(\kappa) \delta\left(\kappa-\kappa^{\prime}\right), \\
& \sum_{n}\left(E^{-1} P\right)_{2 m, 2 n+1} v_{2 n+1}^{-}(\kappa)=p_{e o}(\kappa) v_{2 m}^{-}(\kappa) \\
& \Longrightarrow \sum_{m, n}\left(v^{-}\left(\kappa^{\prime}\right) E\right)_{2 m}\left(E^{-1} P\right)_{2 m, 2 n+1} v_{2 n+1}^{-}(\kappa)=\frac{1}{2} p_{e o}(\kappa) \delta\left(\kappa-\kappa^{\prime}\right) .
\end{aligned}
$$

Similar arguments hold for the eigenvalues $m_{e o}$ and $m_{o e}$ of $(E M)_{m n}$. Then, we can rewrite the squeezed state as

$$
\langle S|=\langle\downarrow| \exp \left[\frac{1}{2} \int_{0}^{\infty} d \kappa\left(\vec{\psi}_{\kappa}^{+} \cdot S(\kappa) \cdot \vec{\psi}_{\kappa}^{-}-\mathrm{i} P_{e o}(\kappa) \psi_{e, \kappa}^{+} \psi_{o, \kappa}^{+}-\mathrm{i} M_{e o}(\kappa) \psi_{e, \kappa}^{-} \psi_{o, \kappa}^{-}\right)\right] .
$$

\footnotetext{
${ }^{10}$ Here, $E$ is the matrix with components $E_{m n}=n \delta_{m n}$ introduced in section 2.

${ }^{11}$ Note that for consistency, $S_{e e}$ and $S_{o o}$ have to be even functions of $\kappa$, and $S_{e o}$ and $S_{o e}$ have to be odd functions of $\kappa$.
} 
with $S(\kappa)=\left(\begin{array}{cc}S_{e e} & -\mathrm{i} S_{e o} \\ -\mathrm{i} S_{o e} & -S_{o o}\end{array}\right), P_{e o}(\kappa)=p_{o e}(\kappa)-p_{e o}(\kappa)$ and $M_{e o}(\kappa)=m_{o e}(\kappa)-m_{e o}(\kappa)$.

Squeezed state conditions. There is a simple criterion of whether a state (3.11) is a squeezed state. This question is particularly important when it comes to the interpretation of the states in our classification as surface states. To begin with, it should be remarked that a squeezed state includes a nonvanishing constant term from the expansion of the exponential (which in the canonical normalization of the state equals 1). As a consequence, a necessary condition for (3.11) to be a squeezed state is

$$
g(\alpha, \beta, \gamma, \varphi, \eta, \lambda):=f(\alpha, \beta, \gamma, \lambda)-\frac{\lambda R^{2}}{\theta^{2}}-\frac{\varphi R}{\theta}+\frac{\eta R}{\theta} \neq 0 .
$$

If this is the case, we may factorize the state into $g(\alpha, \beta, \gamma, \varphi, \eta, \lambda)$ times a canonically normalized state. The condition for the latter to take the exponential form (4.6) is that the $\psi_{\kappa, e}^{+\dagger} \psi_{\kappa, e}^{-\dagger} \psi_{\kappa, o}^{+\dagger} \psi_{\kappa, o}^{-\dagger}|\downarrow\rangle-$ term in (3.11), normalized appropriately, agrees with (the BPZ conjugate of) the term quartic in the $\psi$ 's from the exponential, i.e., with

$$
\frac{1}{4 R^{2}}\left(-S_{e e} S_{o o}+S_{o e} S_{e o}+P_{e o} M_{e o}\right) \psi_{\kappa, e}^{+\dagger} \psi_{\kappa, e}^{-\dagger} \psi_{\kappa, o}^{+\dagger} \psi_{\kappa, o}^{-\dagger}|\downarrow\rangle
$$

where

$$
\begin{gathered}
S_{e e}=\left(f(\alpha, \beta, \gamma, \lambda)+\frac{\lambda R^{2}}{\theta^{2}}+\frac{\varphi R}{\theta}+\frac{\eta R}{\theta}\right) / g(\alpha, \beta, \gamma, \varphi, \eta, \lambda), \\
S_{o o}=-\left(f(\alpha, \beta, \gamma, \lambda)+\frac{\lambda R^{2}}{\theta^{2}}-\frac{\varphi R}{\theta}-\frac{\eta R}{\theta}\right) / g(\alpha, \beta, \gamma, \varphi, \eta, \lambda), \\
S_{o e}=\left(2 \beta R+\lambda R^{2}\right) /(\theta g(\alpha, \beta, \gamma, \varphi, \eta, \lambda)) \\
S_{e o}=-\left(2 \gamma R+\lambda R^{2}\right) /(\theta g(\alpha, \beta, \gamma, \varphi, \eta, \lambda)) \\
M_{e o}=2 \delta R /(\theta g(\alpha, \beta, \gamma, \varphi, \eta, \lambda)) \\
P_{e o}=2 \varepsilon R /(\theta g(\alpha, \beta, \gamma, \varphi, \eta, \lambda))
\end{gathered}
$$

from (3.11). This yields the necessary and sufficient condition

$$
\alpha \lambda=\varphi \eta+\beta \gamma-\delta \varepsilon
$$

a state satisfying this condition (and with $g \neq 0$ ) is a squeezed state of the form (4.6). For a projector state $|P\rangle_{\kappa}$ satisfying eq. (3.6h) this condition simplifies to

$$
0=\lambda(4 f(\alpha, \beta, \gamma, \lambda)-1) \quad \Longrightarrow \quad \lambda=0 \vee f(\alpha, \beta, \gamma, \lambda)=\frac{1}{4}
$$

It may serve as a simple check of (4.6) and (4.9) that for $\alpha=1$, all other parameters zero, we obtain the identity state

$$
\langle\mathcal{I}|=\langle\downarrow| \exp \left(\frac{1}{2} \int_{0}^{\infty} d \kappa \vec{\psi}_{\kappa}^{+} \cdot \vec{\psi}_{\kappa}^{-}\right)
$$

if we take the tensor product over all $\kappa$.

Imposing conditions (4.7) and (4.11) in addition to the classification of real projectors in the last section reduces the number of solutions in some cases:

(I"): $2 \alpha+R \beta+R \gamma=1$

(a"): $\delta \neq 0 \vee \varepsilon \neq 0 \Rightarrow \lambda=0, \delta \varepsilon=\beta \gamma+\varphi \eta=\frac{1}{R^{2}}\left(\alpha^{2}-\alpha\right), \varphi \neq \eta+\frac{\theta}{2 R}, \operatorname{Re} \beta=\operatorname{Re} \gamma=0$.

$\left(b^{\prime \prime}\right)$ : empty.

(II"): $\beta=\gamma$ 


$$
\begin{aligned}
& \text { (a"): } \varphi \neq 0 \vee \eta \neq 0 \Rightarrow \alpha=\frac{1}{2}-R \gamma, \delta \varepsilon=\gamma^{2}-\frac{1}{4 R^{2}}, \varphi \eta=-\frac{1}{4 R^{2}} . \\
& \text { (a1"): } \delta \varepsilon \neq 0 \Rightarrow \lambda=0, \varphi \neq \eta+\frac{\theta}{2 R}, \operatorname{Re} \beta=\operatorname{Re} \gamma=0 . \\
& \text { (a2"): empty. } \\
& \text { (a3"): } \alpha=0, \gamma=\frac{1}{2 R}, \lambda=-\frac{1}{R^{2}}, \varphi \neq \eta+\frac{\theta}{4 R}+\frac{1}{\theta R} . \\
& \text { (b"): } \varphi=\eta=0 \\
& \text { (b1"): } \delta \varepsilon \neq 0 \Rightarrow(\alpha, \gamma, \lambda) \in\left\{\left(\frac{1}{2},-\frac{1}{2 R}, \frac{1}{R^{2}}\right),\left(-\frac{1}{2}, \frac{3}{2 R},-\frac{3}{R^{2}}\right)\right\} . \\
& \text { (b2"): empty. } \\
& \text { (b3"): } \delta=\varepsilon=0 \Rightarrow \alpha=1, \gamma=0, \lambda=0 .
\end{aligned}
$$

It is remarkable that there is only one class (namely (IIa3")) of real neutral (i. e., with $\delta=\varepsilon=0$ ) squeezed state projectors apart from the identity (4.12) in (IIb3"). It will be demonstrated in the next paragraph that this class contains the generalized butterfly states. We will enlarge on this one-parameter family in the next section.

Generalized butterfly states as Moyal projectors. We will now show that the best-known class of surface state projectors, the generalized butterfly states, are contained in the above classification. This family [17] is parametrized by a parameter $a \in[0,2]$, and in the continuous basis all members are proportional to [19]

$$
\left\langle B_{a}\right|=\langle\downarrow| \exp \left(-\frac{1}{2} \int_{0}^{\infty} d \kappa \vec{\psi}_{\kappa}^{+}\left(\begin{array}{cc}
\frac{2 \tanh \left(\frac{\pi \kappa(2-a)}{4 a}\right)-\theta(\kappa)}{2 \tanh \left(\frac{\pi \kappa(2-a)}{4 a}\right)+\theta(\kappa)} & 0 \\
0 & \frac{2 \operatorname{coth}\left(\frac{\pi \kappa(2-a)}{4 a}\right)-\theta(\kappa)}{2 \operatorname{coth}\left(\frac{\pi \kappa(2-a)}{4 a}\right)+\theta(\kappa)}
\end{array}\right) \vec{\psi}_{\kappa}^{-}\right) .
$$

The sliver is recovered in the limit $a \rightarrow 0$; the canonical butterfly state is obtained for $a=1$, and the limit $a \rightarrow 2$ gives the so-called nothing state. A straightforward computation (to be described below) yields that this family of states is given by $\alpha=0, \beta=\gamma=\frac{1}{2 R}, \delta=\varepsilon=0, \eta=\frac{1}{2 h_{a} R}$, $\varphi=-\frac{h_{a}}{2 R}$, and $\lambda=-\frac{1}{R^{2}}$ with

$$
h_{a}(\kappa)=\tanh \left(\frac{\pi \kappa(2-a)}{4 a}\right)
$$

in our classification scheme. Hence, these projectors belong to the subclass (IIa3").

Relation to bosonic projectors. It is interesting to note that there is a one-to-one correspondence between (neutral) squeezed state projectors of our fermionic first order system and those of the bosonic CFT in momentum basis. Namely, an inspection of the corresponding squeezed state formulas yields that a fermionic projector with coefficient matrix $S(\kappa)$ leads to a bosonic projector with the same coefficient matrix (and vice versa). ${ }^{12}$ In the light of this observation, it is not surprising that all our (neutral) squeezed state projectors, i.e., those from (IIa3"), identically fulfill the projector condition of [19], eq. (3.55). Thus, the analysis in other representations (such as the half-string or discrete Moyal representations) given in this reference carry over to our fermionic first order system. There is some hope that the above correspondence can be generalized to the case of non-squeezed state projectors.

Inverse transformation to operators. Sometimes it is useful to describe a given squeezed state in terms of operators under the Moyal-Weyl map, e. g., for the computation of star products or for testing whether it is contained in our above classification (and thus a projector). For simplicity, we

\footnotetext{
${ }^{12}$ In the discrete basis, a fermionic squeezed state projector with coefficients $S_{m n}^{\text {ferm. }}$ maps to a bosonic squeezed state projector with coefficients $S_{m n}^{\text {bos. }}=\frac{1}{2} \sqrt{\frac{n}{m}} S_{m n}^{\text {ferm. }}$.
} 
restrict to uncharged states (with $M_{e o}=P_{e o}=0$ ); however, the derivation can be trivially expanded to the general case. After giving the appropriate formulas, we will describe this procedure for the example of the sliver state.

Instead of solving eq. (4.9) for $\alpha, \ldots, \lambda$, it is much more convenient to transform the squeezed state projector (of the form (4.6)) under consideration to a string functional. This in general leads to a string field of the form

$$
\Psi_{S}[\vec{x}(\kappa), \vec{y}(\kappa)]=\left(\prod_{\kappa} f(\alpha, \beta, \gamma, \lambda)\right) \exp \left(\frac{\mathrm{i}}{2} \int d \kappa \vec{x}(\kappa) \cdot T(\kappa) \cdot \vec{y}(\kappa)\right),
$$

where $T(\kappa)=\left(\begin{array}{cc}T_{e e} & T_{e o} \\ T_{o e} & T_{e e}\end{array}\right)=\left(\frac{S(\kappa)-\mathbb{1}}{S(\kappa)+\mathbb{1}}\right)^{t}$, and the proportionality factor was fixed by comparison with eq. (3.8). Then, expanding the exponential and comparing the coefficients with (3.8) yields

$$
\begin{aligned}
\beta & =\frac{f(\alpha, \beta, \gamma, \lambda) \theta(\kappa)}{R}\left(\frac{1}{4} \operatorname{det} T+\mathrm{i} T_{e o}\right), \\
\gamma & =\frac{f(\alpha, \beta, \gamma, \lambda) \theta(\kappa)}{R}\left(\frac{1}{4} \operatorname{det} T-\mathrm{i} T_{o e}\right), \\
\varphi & =\frac{f(\alpha, \beta, \gamma, \lambda) \theta(\kappa)}{R} T_{e e}, \\
\eta & =-\frac{f(\alpha, \beta, \gamma, \lambda) \theta(\kappa)}{R} T_{o o} \\
\lambda & =-\frac{f(\alpha, \beta, \gamma, \lambda) \theta(\kappa)^{2}}{R^{2}} \operatorname{det} T .
\end{aligned}
$$

These five equations for the four components of $T$ are consistent only if the squeezed state condition (4.10) holds. From the definition (3.12), we finally read off

$$
\alpha=f(\alpha, \beta, \gamma, \lambda)\left(1+\frac{\mathrm{i} \theta(\kappa)}{2}\left(T_{o e}-T_{e o}\right)-\frac{\theta^{2}}{4} \operatorname{det} T\right) .
$$

For squeezed state projectors, the overall scale $f(\alpha, \beta, \gamma, \lambda)$ is fixed to be $\frac{1}{4}$ if $\operatorname{det} T \neq 0$. In all other cases, it has to be determined from the exact normalization of the string field (4.15).

Let us now demonstrate the above procedure in the case of the sliver, i. e., the generalized butterfly state with $a \rightarrow 0$. Since in this limit, $h_{a} \rightarrow 1$, we obtain from (4.13) the ket state (w.r.t. the canonically normalized star product)

$$
|\Xi\rangle=\exp \left[-\delta(0) \int d \kappa \ln \left(\frac{4 \theta^{2}(\kappa)}{(\theta(\kappa)+2)^{2}}\right)\right] \exp \left[-\frac{1}{2} \int_{0}^{\infty} d \kappa \frac{\theta(\kappa)-2}{\theta(\kappa)+2} \vec{\psi}_{\kappa}^{+\dagger} \cdot \vec{\psi}_{\kappa}^{-\dagger}\right]|\downarrow\rangle .
$$

If we contract this with the position eigenstate (2.25) the string field functional for the sliver turns out to be

$$
\Psi_{\Xi}(\vec{x}(\kappa), \vec{y}(\kappa))=\langle\vec{X} \mid \Xi\rangle \propto \exp \left[-\mathrm{i} \int_{0}^{\infty} d \kappa \frac{\vec{x}(\kappa) \cdot \vec{y}(\kappa)}{\theta(\kappa)}\right] .
$$

This leads to the values $\alpha=0, \beta=\gamma=\frac{2 f}{R}, \varphi=-\frac{2 f}{R}, \eta=\frac{2 f}{R}$ as well as $\lambda=-\frac{4 f}{R^{2}}$. The squeezed state condition requires $f=\frac{1}{4}$ in accordance with the discussion on generalized butterflies (the proportionality factor is an infinite product as in (4.15)). 


\section{$5 \quad$ Identification of surface states}

Some of the projectors of our classification scheme described in the preceding section can be identified with well-known surface states. Even though this identification is rather technical in general, it is possible and will be done for some cases in this section.

To begin with, we note that surface states have a representation as exponentials of (linear combinations of) Virasoro generators, i. e., we can limit our search for surface states to squeezed states of the form (4.6). However, not all such states are eligible, namely states with $P_{m n} \neq 0$ or $M_{m n} \neq 0$ cannot be expressed in terms of (neutral) Virasoro generators. This restricts our search to the one-parameter class given by

$$
\alpha=0, \quad \beta=\gamma=\frac{1}{2 R}, \quad \delta=\varepsilon=0, \quad \eta=\frac{1}{2 r R}, \quad \varphi=-\frac{r}{2 R}, \quad \text { and } \quad \lambda=-\frac{1}{R^{2}},
$$

where $r(\kappa)$ is a free odd function of $\kappa$ (the latter requirement ensures that $S_{e e}$ and $S_{o o}$ are even functions). All projectors in this class have diagonal matrices $S$, i. e., $S_{e o}=S_{o e}=0$. This class also contains the generalized butterfly states for the special choice $r=h_{a}$, cf. section 3 .

General method. A surface state $\left\langle\Sigma^{f}\right|$ is determined by a map $f: H \rightarrow \Sigma$ from the canonical upper half disk $\{|z| \leq 1\} \subset \mathbb{C}$ onto a Riemann surface $\Sigma$ with boundary and the requirement that

$$
\left\langle\Sigma^{f} \mid \phi\right\rangle=\langle f \circ \phi(0)\rangle_{\Sigma}
$$

for all Fock space states $|\phi\rangle$ of weight $h$ in the boundary CFT with corresponding operators $\phi(z)$. The correlation function \langle\rangle$_{\Sigma}$ is evaluated on $\Sigma$, and $f \circ \phi(0)=\left(f^{\prime}(0)\right)^{h} \phi(f(0))$ is the conformal transform of $\phi$ by the map $f$.

Now, let us evaluate the correlation function (5.2) for a surface state of the form (4.1) with $M_{m n}=$ $P_{m n}=0$ and for $|\phi\rangle=\psi^{+}(z) \psi^{-}(w) \psi^{+}(0)|\downarrow\rangle,{ }^{13}$

$$
\left\langle\downarrow\left|\exp \left[\frac{1}{2} \sum_{m, n>0} \psi_{m}^{+} S_{m n} \psi_{n}^{-}\right] \sum_{k=-\infty}^{\infty} \frac{\psi_{k}^{+}}{z^{k}} \sum_{l=-\infty}^{-1} \frac{\psi_{l}^{-}}{w^{l+1}}\right| \downarrow\right\rangle=2 \sum_{m, n>0} S_{m n} z^{n} w^{m-1}+\frac{2}{z-w} \frac{z}{w} .
$$

On the other hand, according to eq. (5.2), this correlation function should be equal to ${ }^{14}$

$$
\langle f \circ \phi\rangle_{\Sigma}=\frac{2 f^{\prime}(w)}{f(z)-f(w)} \frac{f(z)-f(0)}{f(w)-f(0)} ;
$$

and we can use $S L(2, \mathbb{R})$ invariance to fix $f(0)=0, f^{\prime}(0)=1$, and $f^{\prime \prime}(0)=0$. Then, (one half of) the nonsingular part of the correlator becomes [56, 47]

$$
S(z, w):=\sum_{m, n>0} S_{m n} z^{n} w^{m-1}=\frac{f^{\prime}(w)}{f(z)-f(w)} \frac{f(z)}{f(w)}-\frac{1}{z-w} \frac{z}{w},
$$

therefore, deriving w.r.t. $z$ and choosing $w=0$,

$$
\frac{\partial}{\partial z} S(z, 0)=-\frac{f^{\prime}(z)}{f(z)^{2}}+\frac{1}{z^{2}}
$$

\footnotetext{
${ }^{13}$ The $U(1)$ charges of the insertions of the correlation functions have to sum up to +1 in order to give a nonvanishing result. - The somewhat unusual factors of 2 in the next two formulas stem from our normalization (2.5).

${ }^{14}$ Recall that the second factor originates from the nontrivial background charge of the system [36].
} 
This equation can be integrated to give

$$
f(z)=\frac{z}{z S(z, 0)+1}
$$

for a candidate function $f$ for the map defining the surface state $\left\langle S^{f}\right|$. Here, the integration constant was chosen in such a way that for $\left\langle S^{f}\right|=\langle\downarrow|$, we obtain the identity map $f(z)=z$.

Obviously, the defining map $f(z)$ is encoded in the coefficients $S_{m n}$ via (5.5); we can extract a candidate function with the help of eq. (5.7). From our classification of the projectors in the last section, we know the $S_{m n}$ in terms of the eigenvalues $S_{e e}, S_{o e}, S_{e o}$, and $S_{o o}$. Since all squeezed state projectors under consideration have $S_{e o}=S_{o e}=0$, in fact only $S_{e e}$ and $S_{o o}$ contribute. For the choice of parameters given in (5.1) these eigenvalues are parametrized by

$$
S_{e e}(\kappa)=-1+\frac{\theta(\kappa)}{\theta(\kappa)+2 r(\kappa)}, \quad S_{o o}(\kappa)=-1+\frac{4}{2+r(\kappa) \theta(\kappa)} .
$$

Now, $S(z, w)$ can be reconstructed from these eigenvalues using (2.12), (2.13), and (4.4),

$$
S(z, w)=\frac{2}{w} \int_{0}^{\infty} d \kappa\left[f_{v_{e}^{+}}(\kappa, w) f_{v_{e}^{-}}(\kappa, z) S_{e e}(\kappa)+f_{v_{o}^{+}}(\kappa, w) f_{v_{o}^{-}}(\kappa, z) S_{o o}(\kappa)\right] .
$$

It should be noted that $f(z)$ is purely given by the $S_{o o}$-part, see (5.7) and

$$
S(z, 0)=\int_{0}^{\infty} d \kappa \frac{\sinh \kappa Z}{\sinh \frac{\pi \kappa}{2}} S_{o o}(\kappa) .
$$

As in section 2, $Z$ abbreviates $\tan ^{-1} z$. Given a function $r(\kappa)$ such that the integral (5.9) converges, we can determine a map $f(z)$ via (5.7). Obviously, $f$ is an odd function mapping the upper unit half-disk into some region of the upper half-plane.

Consistency conditions for surface states. We will now dicuss two consistency conditions on a map $f(z)$ obtained in this way:

If the map $f(z)$ defines a surface state projector, eqs. (5.5) and (5.9) necessarily have to agree (in fact, this condition is also sufficient [19]). Since $f(z)$ is constructed solely from the $S_{o o}$-part in (5.9) and $S(z, w)$ as given by (5.9) also contains $S_{e e}$, this is a restriction on the function $r$ in (5.8). However, we found it difficult to solve this constraint for $r(\kappa)$ (e.g., by variational methods) due to the nonlinearity of the right-hand side of the CFT expression (5.5) in $S_{o o}$.

Instead, it is possible to derive a second (necessary) consistency condition for $f(z)$ which has to hold for any surface state projector diagonal in the $\kappa$-basis and which can be used to determine the form of possible $f$ 's. The starting point is the observation that the $S_{o o}$-part of eq. (5.9) is given by $S(z, 0)$ and the $S_{e e}$-part can be determined via (an integral of) $\left.\frac{\partial}{\partial w} S(z, w)\right|_{w=0}$. Thus, the full integral (5.9) can be computed via $f(z)$. Together with (5.5), this gives a restriction on $f(z)$.

Firstly, note that (5.9) can be rewritten as

$$
S(z, w)=\frac{1}{1+w^{2}}\left[-S_{1}(W)+\frac{1}{2} S_{1}(Z+W)-\frac{1}{2} S_{1}(Z-W)+\frac{1}{2} S_{2}(Z+W)+\frac{1}{2} S_{2}(Z-W)\right]
$$

again with $W=\tan ^{-1} w, Z=\tan ^{-1} z$, and

$$
S_{1}(Z)=\int_{0}^{\infty} d \kappa \frac{\sinh \kappa Z}{\sinh \frac{\pi \kappa}{2}} S_{e e}(\kappa), \quad S_{2}(Z)=\int_{0}^{\infty} d \kappa \frac{\sinh \kappa Z}{\sinh \frac{\pi \kappa}{2}} S_{o o}(\kappa) .
$$


Obviously, $S_{2}\left(\tan ^{-1} z\right)=\frac{1}{f(z)}-\frac{1}{z}$.

Next, it is easy to see that due to the form of (5.9), only the $S_{e e}$-part will contribute to $\tilde{S}(Z):=\left.\frac{\partial}{\partial w}\left(1+w^{2}\right) S(z, w)\right|_{w=0}=\left.\frac{\partial}{\partial w} S(z, w)\right|_{w=0}$; in addition, one can recover $S_{1}(Z)$ fully from $\int_{0}^{Z} d Z^{\prime}\left(\tilde{S}\left(Z^{\prime}\right)+\tilde{S}_{0}\right)$ with some additive constant $\tilde{S}_{0}$. Eq. (5.11) ensures that the contributions of this constant to $S(z, w)$ cancel.

Following this prescription, we have to compute $\left.\frac{\partial}{\partial w} S(z, w)\right|_{w=0}$ from (5.5). A direct computation with our choice $f(0)=0, f^{\prime}(0)=1, f^{\prime \prime}(0)=0$ leads to a difference of singularities. In order to avoid this one can compute $\frac{\partial^{2}}{\partial z \partial w} S(z, w)$, set $w=0$, and integrate with respect to $z$. If one absorbs the integration constant into $\tilde{S}_{0}$, the result is

$$
\left.\frac{\partial}{\partial w} S(z, w)\right|_{w=0}=\frac{1}{f(z)^{2}}-\frac{1}{z^{2}} \quad \Longrightarrow \quad \tilde{S}(Z)=\frac{1}{F(Z)^{2}}-\frac{1}{\tan ^{2} Z}
$$

with $F(Z):=f(\tan Z)=f(z)$. Putting everything together, we obtain from (5.11):

$$
\begin{aligned}
\left(1+w^{2}\right) S(z, w)= & -\int_{0}^{W} d Z^{\prime} \tilde{S}\left(Z^{\prime}\right)+\frac{1}{2} \int_{0}^{Z+W} d Z^{\prime} \tilde{S}\left(Z^{\prime}\right)-\frac{1}{2} \int_{0}^{Z-W} d Z^{\prime} \tilde{S}\left(Z^{\prime}\right) \\
& +\frac{1}{2}\left(\frac{1}{F(Z+W)}-\frac{1}{\tan (Z+W)}\right)+\frac{1}{2}\left(\frac{1}{F(Z-W)}-\frac{1}{\tan (Z-W)}\right) .
\end{aligned}
$$

This expression should be compared with eq. (5.5). Finally, a somewhat messy calculation leads to the following result:

$$
\frac{1}{2} \frac{1-F^{\prime}(Z+W)}{F(Z+W)^{2}}+\frac{1}{2} \frac{1+F^{\prime}(Z-W)}{F(Z-W)^{2}}=\frac{F^{\prime \prime}(W)}{F(W)}+\frac{1-F^{\prime}(W)^{2}}{F(W)^{2}}+\frac{F^{\prime \prime}(W)}{F(Z)-F(W)}+\frac{F^{\prime}(W)^{2}}{(F(Z)-F(W))^{2}} .
$$

This condition restricts the allowed maps $f(z)$. A short computation shows that it is indeed satisfied for the generalized butterfly states with $F(Z)=\frac{1}{a} \sin a Z$ as well as the identity state with $F(Z)=\frac{1}{2} \tan 2 Z .^{15}$ By a differentiation w.r.t. $Z$ and an integration over $W$, eq. (5.15) can be transformed into

$$
-\frac{F^{\prime}(Z) F^{\prime}(W)}{(F(Z)-F(W))^{2}}=\frac{1-F^{\prime}(Z+W)}{2 F(Z+W)^{2}}-\frac{1+F^{\prime}(Z-W)}{2 F(Z-W)^{2}} .
$$

The integration constant is fixed by the initial conditions $F(0)=0, F^{\prime}(0)=1$, and $F^{\prime \prime}(0)=0$. In this formulation, the left-hand side of eq. (5.15) is a bosonic two-point function. It is easy to see that condition (5.16) is a refined version of the surface state condition (3.40) in [19], cf. also eq. (3.30) in this reference.

Generalized butterfly states. Exemplarily, we will demonstrate the method in the case of the butterfly states (4.13) which are known to be surface states. The integral we have to compute is

$$
S(z, 0)=\int_{0}^{\infty} d \kappa \frac{\sinh \kappa Z}{\sinh \frac{\pi \kappa}{2}} \frac{2 \operatorname{coth}\left(\frac{\pi \kappa(2-a)}{4 a}\right)-\theta(\kappa)}{2 \operatorname{coth}\left(\frac{\pi \kappa(2-a)}{4 a}\right)+\theta(\kappa)} .
$$

Decomposing $\operatorname{coth}\left(\frac{\pi \kappa(2-a)}{4 a}\right)=\frac{2-\tanh \left(\frac{\pi \kappa}{2 a}\right) \theta(\kappa)}{2 \tanh \left(\frac{\pi \kappa}{2 a}\right)-\theta(\kappa)}$, we obtain

$$
S(z, 0)=\int_{0}^{\infty} d \kappa \frac{\sinh \kappa Z}{\sinh \frac{\pi \kappa}{2}}\left(1-\frac{\theta(\kappa)-2 \tanh \left(\frac{\pi \kappa}{2 a}\right)}{\frac{\theta(\kappa)}{2}-\frac{2}{\theta(\kappa)}}\right) .
$$

\footnotetext{
${ }^{15}$ In fact, this relation also holds for all wedge states with $F(Z)=\frac{1}{a} \tan a Z$ which can even be obtained by an integration as in (5.7) and (5.10). However, the necessary and sufficient condition that eqs. (5.5) and (5.9) have to agree is only satisfied for the identity and butterfly states.
} 
The first part can immediately be integrated with the help of

$$
\int_{0}^{\infty} d x \frac{\sinh a x}{\sinh b x}=\frac{\pi}{2 b} \tan \frac{\pi a}{2 b} \quad \text { for } \quad|\operatorname{Re} a|<\operatorname{Re} b .
$$

Using $\frac{\theta(\kappa)}{2}-\frac{2}{\theta(\kappa)}=-2 \frac{1}{\sinh \left(\frac{\pi \kappa}{2}\right)}$, we thus have to evaluate

$$
\begin{aligned}
S(z, 0) & =z+\int_{0}^{\infty} d \kappa \sinh (\kappa Z)\left(\tanh \left(\frac{\pi \kappa}{4}\right)-\tanh \left(\frac{\pi \kappa}{2 a}\right)\right) \\
& =z+\int_{1 / a}^{1 / 2} d y \int_{0}^{\infty} d \kappa \frac{\sinh (\kappa Z)}{\cosh \left(\frac{\pi \kappa y}{2}\right)^{2}} \frac{\pi \kappa}{2}
\end{aligned}
$$

where we have rewritten the difference of the two tanh's as an integral over the derivative of the tanh. In order to get rid of the simple power of $\kappa$ in the integrand, we rewrite the latter as a derivative, so that we obtain the Gradshteyn/Ryzhik-integrable expression [57]

$$
\begin{aligned}
S(z, 0) & =z+\frac{\pi}{2} \frac{\partial}{\partial Z} \int_{1 / a}^{1 / 2} d y \int_{0}^{\infty} d \kappa \frac{\cosh (\kappa Z)}{\cosh \left(\frac{\pi \kappa y}{2}\right)^{2}} \\
& =z+\frac{\partial}{\partial Z} \int_{1 / a}^{1 / 2} d y \frac{\frac{Z}{y^{2}}}{\sin \frac{Z}{y}} .
\end{aligned}
$$

But since for a function $F\left(\frac{Z}{y}\right), \frac{1}{y} \frac{\partial}{\partial Z} F\left(\frac{Z}{y}\right)=-\frac{1}{Z} \frac{\partial}{\partial y} F\left(\frac{Z}{y}\right)$, the $y$-integration is trivial to do, and $S(z, 0)$ simplifies to

$$
S(z, 0)=z+\frac{a}{\sin a Z}-\frac{2}{\sin 2 Z}=-\frac{1}{z}+\frac{a}{\sin a Z} .
$$

This yields the well-known expression

$$
f(z)=\frac{1}{a} \sin \left(a \tan ^{-1} z\right)
$$

for the map defining the generalized butterfly states.

\section{Conclusions}

In this paper we have considered star algebra projectors for a fermionic first order system of weights $(1,0)$. To this end we have formulated the interaction vertex in the continuous Moyal basis. In this basis the oscillators form two Clifford algebras labeled by $\kappa \in \mathbb{R}$. We have classified all projectors which factorize into projectors for each value of $\kappa$ by employing a fermionic version of the MoyalWeyl map known from noncommutative field theories. BPZ-real squeezed state projectors which are neutral w.r.t. the $U(1)$ (ghost number) current turn out to be naturally parametrized by a single odd and integrable function of the parameter $\kappa$. We have shown that this class of projectors contains the generalized butterfly states as a subclass. A method how to determine surface states in this class of projectors is given and a condition on the maps defining the shape of the surface state is derived.

At least for the case of squeezed state projectors described above one can recover the full set of bosonic projectors. It is tempting to believe that this extends to non-squeezed state projectors which are neutral w.r.t. the $U(1)$ (ghost) current as well. 
It would be interesting to solve the consistency condition on the maps defining surface states. Those solutions which indeed can be obtained from integrations as in (5.9) and (5.10) will correspond to surface state projectors which are diagonal in the $\kappa$-basis. It would be fascinating to see whether the generalized butterfly states and the identity state are the only solutions, and if not, whether it is possible to obtain surface state projectors without the singular property that the boundary of the surface touches the midpoint. This is indeed the case for the identity, but no finite rank projector with this property is known. Moreover, it would be worthwhile to study the projectors we have classified in a continuous half string basis along the lines of [58].

Another remarkable result we have obtained is that the normalization factors from rewriting the star product in the Moyal basis cancel between bosons and fermions in any even dimension. This is a consequence of the close relation of the corresponding Neumann coefficients. Hence, up to an overall normalization factor the star product of $\mathrm{N}=2$ string field theory is a canonically normalized continuous tensor product of Moyal-Weyl products. This is a distinguished feature of this type of string field theory.

\section{Acknowledgements}

SU would like to thank Martin Schnabl and Yuji Okawa for discussions. This work was done within the framework of the DFG priority program "String Theory" (SPP 1096). The work of SU was supported by a fellowship within the postdoc program of the German Academic Exchange Service (DAAD).

\section{References}

[1] K. Ohmori, A review on tachyon condensation in open string field theories, arXiv:hepth/0102085.

[2] P. J. De Smet, Tachyon condensation: Calculations in string field theory, arXiv:hepth/0109182.

[3] I. Ya. Aref'eva, D. M. Belov, A. A. Giryavets, A. S. Koshelev and P. B. Medvedev, Noncommutative field theories and (super)string field theories, arXiv:hep-th/0111208.

[4] W. Taylor, Lectures on D-branes, tachyon condensation, and string field theory, arXiv:hepth/0301094.

[5] W. Taylor and B. Zwiebach, D-branes, tachyons, and string field theory, arXiv:hep-th/0311017.

[6] E. Witten, Noncommutative geometry and string field theory, Nucl. Phys. B 268 (1986) 253.

[7] E. Witten, Interacting field theory of open superstrings, Nucl. Phys. B 276 (1986) 291.

[8] N. Berkovits, Super-Poincaré invariant superstring field theory, Nucl. Phys. B 450 (1995) 90, Erratum ibid. B 459 (1996) 439, [arXiv:hep-th/9503099].

[9] L. Rastelli, A. Sen and B. Zwiebach, String field theory around the tachyon vacuum, Adv. Theor. Math. Phys. 5 (2002) 353 [arXiv:hep-th/0012251].

[10] L. Rastelli, A. Sen and B. Zwiebach, Half strings, projectors, and multiple D-branes in vacuum string field theory, JHEP 0111 (2001) 035 [arXiv:hep-th/0105058]. 
[11] D. J. Gross and W. Taylor, Split string field theory. I, JHEP 0108 (2001) 009 [arXiv:hepth/0105059].

[12] L. Rastelli, A. Sen and B. Zwiebach, Boundary CFT construction of D-branes in vacuum string field theory, JHEP 0111 (2001) 045 [arXiv:hep-th/0105168].

[13] D. J. Gross and W. Taylor, Split string field theory. II, JHEP 0108 (2001) 010 [arXiv:hepth/0106036].

[14] G. Moore and W. Taylor, The singular geometry of the sliver, JHEP 0201 (2002) 004 [arXiv:hep-th/0111069].

[15] M. Mariño and R. Schiappa, Towards vacuum superstring field theory: The supersliver, J. Math. Phys. 44 (2003) 156 [arXiv:hep-th/0112231].

[16] M. Schnabl, Anomalous reparametrizations and butterfly states in string field theory, Nucl. Phys. B 649 (2003) 101 [arXiv:hep-th/0202139].

[17] D. Gaiotto, L. Rastelli, A. Sen and B. Zwiebach, Star algebra projectors, JHEP 0204 (2002) 060 [arXiv:hep-th/0202151].

[18] I. Ya. Aref'eva, A. A. Giryavets and A. S. Koshelev, NS ghost slivers, Phys. Lett. B 536 (2002) 138 [arXiv:hep-th/0203227].

[19] E. Fuchs, M. Kroyter and A. Marcus, Squeezed state projectors in string field theory, JHEP 0209 (2002) 022 [arXiv:hep-th/0207001].

[20] D. Mamone, Interpolating state in string field theory, arXiv:hep-th/0311204.

[21] V. A. Kostelecký and R. Potting, Analytical construction of a nonperturbative vacuum for the open bosonic string, Phys. Rev. D 63 (2001) 046007 [arXiv:hep-th/0008252].

[22] H. Hata and T. Kawano, Open string states around a classical solution in vacuum string field theory, JHEP 0111 (2001) 038 [arXiv:hep-th/0108150].

[23] D. Gaiotto, L. Rastelli, A. Sen and B. Zwiebach, Ghost structure and closed strings in vacuum string field theory, Adv. Theor. Math. Phys. 6 (2003) 403 [arXiv:hep-th/0111129].

[24] Y. Okawa, Some exact computations on the twisted butterfly state in string field theory, arXiv:hep-th/0310264.

[25] L. Bonora, C. Maccaferri and P. Prester, Dressed sliver solutions in vacuum string field theory, arXiv:hep-th/0311198.

[26] Y. Okawa, Solving Witten's string field theory using the butterfly state, arXiv:hep-th/0311115.

[27] O. Lechtenfeld and A. D. Popov, On the integrability of covariant field theory for open $N=2$ strings, Phys. Lett. B 494 (2000) 148 [arXiv:hep-th/0009144]

[28] O. Lechtenfeld, A. D. Popov and S. Uhlmann, Exact solutions of Berkovits' string field theory, Nucl. Phys. B 637 (2002) 119 [arXiv:hep-th/0204155].

[29] A. Kling, O. Lechtenfeld, A. D. Popov and S. Uhlmann, On nonperturbative solutions of superstring field theory, Phys. Lett. B 551 (2003) 193 [arXiv:hep-th/0209186]. 
[30] A. Kling, O. Lechtenfeld, A. D. Popov and S. Uhlmann, Solving string field equations: New uses for old tools, Fortschr. Phys. 51 (2003) 775 [arXiv:hep-th/0212335].

[31] T. Kawano and K. Okuyama, Open string fields as matrices, JHEP 0106 (2001) 061 [arXiv:hep-th/0105129].

[32] A. LeClair, M. E. Peskin and C. R. Preitschopf, String field theory on the conformal plane. 1. Kinematical principles, Nucl. Phys. B 317 (1989) 411.

[33] A. LeClair, M. E. Peskin and C. R. Preitschopf, String field theory on the conformal plane. 2. Generalized gluing, Nucl. Phys. B 317 (1989) 464.

[34] N. Berkovits and W. Siegel, Covariant field theory for self-dual strings, Nucl. Phys. B 505 (1997) 139 [arXiv:hep-th/9703154].

[35] D. Friedan, E. J. Martinec and S. H. Shenker, Conformal invariance, supersymmetry and string theory, Nucl. Phys. B 271 (1986) 93.

[36] A. Kling and S. Uhlmann, String field theory vertices for fermions of integral weight, JHEP 0307 (2003) 061 [arXiv:hep-th/0306254].

[37] C. Maccaferri and D. Mamone, Star democracy in open string field theory, arXiv:hepth/0306252.

[38] M. R. Douglas, H. Liu, G. Moore and B. Zwiebach, Open string star as a continuous Moyal product, JHEP 0204 (2002) 022 [arXiv:hep-th/0202087].

[39] D. M. Belov, Diagonal representation of open string star and Moyal product, arXiv:hepth/0204164.

[40] I. Ya. Aref'eva and A. A. Giryavets, Open superstring star as a continuous Moyal product, JHEP 0212 (2002) 074 [arXiv:hep-th/0204239].

[41] T. G. Erler, Moyal formulation of Witten's star product in the fermionic ghost sector, arXiv:hep-th/0205107.

[42] D. M. Belov and A. Konechny, On continuous Moyal product structure in string field theory, JHEP 0210 (2002) 049 [arXiv:hep-th/0207174].

[43] D. M. Belov and C. Lovelace, Witten's vertex made simple, Phys. Rev. D 68 (2003) 066003 [arXiv:hep-th/0304158];

[44] D. M. Belov, Witten's ghost vertex made simple (bc and bosonized ghosts), arXiv:hepth/0308147.

[45] I. Bars, Map of Witten's * to Moyal's *, Phys. Lett. B 517 (2001) 436 [arXiv:hep-th/0106157].

[46] I. Bars and Y. Matsuo, Computing in string field theory using the Moyal star product, Phys. Rev. D 66 (2002) 066003 [arXiv:hep-th/0204260].

[47] I. Bars, I. Kishimoto and Y. Matsuo, Fermionic ghosts in Moyal string field theory, JHEP 0307 (2003) 027 [arXiv:hep-th/0304005].

[48] D. J. Gross and A. Jevicki, Operator formulation of interacting string field theory, Nucl. Phys. B 283 (1987) 1. 
[49] L. Rastelli, A. Sen and B. Zwiebach, Classical solutions in string field theory around the tachyon vacuum, Adv. Theor. Math. Phys. 5 (2002) 393 [arXiv:hep-th/0102112].

[50] L. Rastelli, A. Sen and B. Zwiebach, Star algebra spectroscopy, JHEP 0203 (2002) 029 [arXiv:hep-th/0111281].

[51] E. Fuchs, M. Kroyter and A. Marcus, Virasoro operators in the continuous basis of string field theory, JHEP 0211 (2002) 046 [arXiv:hep-th/0210155].

[52] D. M. Belov and A. Konechny, On spectral density of Neumann matrices, Phys. Lett. B 558 (2003) 111 [arXiv:hep-th/0210169].

[53] K. Okuyama, Ghost kinetic operator of vacuum string field theory, JHEP 0201 (2002) 027 [arXiv:hep-th/0201015];

[54] K. Okuyama, Ratio of tensions from vacuum string field theory, JHEP 0203 (2002) 050 [arXiv:hep-th/0201136].

[55] M. R. Gaberdiel and B. Zwiebach, Tensor constructions of open string theories I: Foundations, Nucl. Phys. B 505 (1997) 569 [arXiv:hep-th/9705038].

[56] T. Okuda, The equality of solutions in vacuum string field theory, Nucl. Phys. B 641 (2002) 393 [arXiv:hep-th/0201149].

[57] I. S. Gradshteyn, I. M. Ryzhik, Table of integrals, series, and products, 5th edition, Academic Press (1994), formulas 3.512.1 and 3.192.1.

[58] E. Fuchs, M. Kroyter and A. Marcus, Continuous half-string representation of string field theory, JHEP 0311 (2003) 039 [arXiv:hep-th/0307148]. 